\title{
From world-sheet supersymmetry to super target spaces
}

\author{
Thomas Creutzig $^{a}$ and Peter B. Rønne $e^{b, c}$ \\ ${ }^{a}$ Department of Physics and Astronomy, University of North Carolina, \\ Phillips Hall, CB 3255, Chapel Hill, NC 27599-3255, U.S.A. \\ ${ }^{b}$ DESY Theory Group, DESY Hamburg \\ Notkestrasse 85, D-22607 Hamburg, Germany \\ ${ }^{c}$ National Institute for Theoretical Physics and Centre for Theoretical Physics, \\ University of the Witwatersrand, \\ Wits, 2050, South Africa \\ E-mail: creutzig@physics.unc.edu, peter.roenne@wits.ac.za
}

ABSTRACT: We investigate the relation between $\mathcal{N}=(2,2)$ superconformal Lie group WZNW models and Lie supergroup WZNW models. The B-twist of an exactly marginal perturbation of the world-sheet superconformal sigma model is the supergroup model. Moreover, the superconformal currents are expressed in terms of Lie superalgebra currents in the twisted theory. As applications, we find protected sectors and boundary actions in the supergroup sigma model. A special example is the relation between string theory on $\mathrm{AdS}_{3} \times \mathrm{S}^{3} \times \mathrm{T}^{4}$ in the RNS formalism and the $\mathrm{U}(1,1 \mid 2) \times \mathrm{U}(1 \mid 1) \times \mathrm{U}(1 \mid 1)$ supergroup WZNW model.

KeYwords: AdS-CFT Correspondence, Boundary Quantum Field Theory, Conformal Field Models in String Theory, Sigma Models

ARXIV EPRINT: 1006.5874 


\section{Contents}

1 Introduction 1

2 Superconformal and supergroup WZNW models 3

2.1 Supersymmetric WZNW models 4

2.2 A Sugawara-like construction of the superconformal algebra 4

2.3 Deformations 6

$\begin{array}{lll}2.4 & \text { Topological conformal field theory } & 7\end{array}$

$\begin{array}{lll}2.5 & \text { Supersymmetric deformations } & 7\end{array}$

$\begin{array}{lll}2.6 & \text { Lie supergroup WZNW models } & 9\end{array}$

3 From world-sheet supersymmetry to supergroups 11

$\begin{array}{lll}3.1 & \text { Some properties of the GL }(\mathrm{N} \mid \mathrm{N}) \text { WZNW model } & 12\end{array}$

3.2 The $\mathcal{N}=(2,2)$ GL $(\mathrm{N}) \times \mathrm{GL}(\mathrm{N})$ WZNW model 13

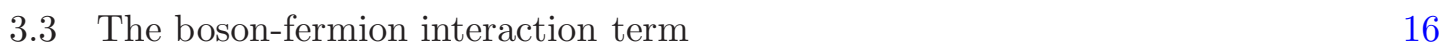

$\begin{array}{ll}3.4 & \text { The principal chiral field as a D-term } \\ \end{array}$

4 Examples and applications $\quad 18$

$\begin{array}{lll}\text { 4.1 GL(2|2) and screening charges as chiral perturbations } & 18\end{array}$

4.2 Comparison to string theory on $\mathrm{AdS}_{3} \times \mathrm{S}^{3} \times \mathrm{T}^{4} \quad 21$

$\begin{array}{lll}4.3 & \text { Boundary actions and the Warner problem } & 25\end{array}$

$\begin{array}{llr}5 & \text { Summary and outlook } & 28\end{array}$

\section{Introduction}

In this note we consider a relation between sigma models on bosonic groups with $\mathcal{N}=(2,2)$ world-sheet supersymmetry and models with supergroups as target spaces via topological twisting. Our motivation to ask for such a relation and to understand it in detail comes from two sides.

The first motivation to study the relation comes from boundary theories on supergroups. For WZNW models on type I Lie supergroups there exists a nice prescription to compute correlation functions in the bulk theory [1-4]. The WZNW model is equivalent to a model consisting of the WZNW model of the bosonic subgroup, free fermions and an interaction term that couples bosons and fermions. The first observation we make, is that the action of the model without the interaction term resembles the topological twist of an $\mathcal{N}=(2,2)$ superconformal field theory. 
Now, we would like to have a similar free fermion prescription for the boundary type I supergroup WZNW model. So far only in the case of GL(1|1) this is known [5]. ${ }^{1}$ There, in addition to the bulk fermions, one had to introduce an additional fermionic boundary degree of freedom. Moreover, the boundary screening charge looks like the square root of the bulk interaction term. These two features are well-known in world-sheet supersymmetric theories, i.e. in order to preserve $\mathcal{N}=2$ superconformal symmetry on the boundary additional boundary fermions plus a factorization of the bulk superpotential into boundary superpotentials is required [7]. We want to understand why we have such a similar behaviour. Moreover, we would like to use techniques from world-sheet supersymmetry to find boundary actions and hence a perturbative description involving free fermions to solve boundary supergroup WZNW models.

The second hint of the relation came from non-trivial exact checks of the $\mathrm{AdS}_{3} / \mathrm{CFT}_{2}$ correspondence $[8,9]$. Here correlation functions of chiral primary operators in the weak coupling limit of string theory on $\mathrm{AdS}_{3} \times \mathrm{S}^{3} \times \mathrm{T}^{4}$ were calculated, and precise agreement was found with calculations done in the dual two-dimensional conformal field theory. Such an agreement is at first sight surprising since the computations in the bulk and on the boundary correspond to different points in moduli space, and some protection of the correlators must be present. In [10] the explanation for the boundary side was given. The argument utilizes that the dual conformal theory has a whole $\mathcal{N}=(4,4)$ worth of supersymmetry. Using this extended supersymmetry, the correlators, which correspond to an $\mathcal{N}=(2,2)$ chiral ring, can be shown to be covariantly constant over the total moduli space.

The question is whether we can now explain this from the string theory side, which only has $\mathcal{N}=(2,2)$ world-sheet supersymmetry, by finding some protected sectors. From [11] we know that string theory on $\mathrm{AdS}_{3} \times \mathrm{S}^{3} \times \mathrm{T}^{4}$ in the hybrid formalism has a description in terms of the PSU $(1,1 \mid 2)$ supergroup sigma model where RR-deformations correspond to deformations away from the WZNW point. This lead us to the search for topological sectors in $\operatorname{PSU}(1,1 \mid 2)$. We, however, only found such sectors in $\mathrm{U}(1,1 \mid 2)$, and in general in $\operatorname{GL}(N \mid N)$. Since a conformal topological sector correspond to the twist of a worldsheet supersymmetric theory, this suggests a relation between the $\mathcal{N}=(2,2)$ world-sheet supersymmetric $\mathrm{GL}(N) \times \mathrm{GL}(N)$ sigma model and the $\mathrm{GL}(N \mid N)$ supergroup model via twisting. Note that it is important that the supergroup has superdimension zero since the world-sheet supersymmetric theory has the same number of bosons and fermions.

Moreover, for an arbitrary supergroup computing all correlation functions is out of reach at the moment. One could thus be less ambitious and restrict to correlators involving fields in a subsector of the theory. A good subsector is then the cohomology of a BRST-like operator $Q$ such that the Lagrangian splits into the Lagrangian of a simpler model plus a $Q$-exact term. The correlators of the cohomology can then be computed in the model corresponding to the simpler Lagrangian [12]. In $\mathcal{N}=(2,2)$ superconformal models such good subsectors are naturally the chiral rings. With the relation presented in this note we then get supergroup analogs of chiral rings, and thus distinguished good sectors of GL(N|N) supergroup models in which we might be able to compute interesting correlation functions.

\footnotetext{
${ }^{1}$ Also for the OSP $(1 \mid 2)$ boundary WZNW model such a free fermion realization is known and used [6].
} 
The Lie supergroup GL(N|N) has various applications in statistical and condensed matter physics, especially in the context of disordered fermion systems [13] and the integer Quantum Hall effect [14]. The supergroup GL(1|1) appeared as a topological twist in the problem of percolation and polymers [15].

What we do in detail is to consider the $\mathcal{N}=(1,1)$ world-sheet supersymmetric version of the $\mathrm{GL}(N) \times \mathrm{GL}(N)$ WZNW model. Using a particular Manin triple decomposition of the algebra we explicitly construct an extension to $\mathcal{N}=(2,2)$ supersymmetry [16]. This extended supersymmetry is preserved under certain deformations of the theory which just add background charges to certain fields. We then perform a B-twist of the theory to obtain a conformal theory which is topological when restricting to the BRST cohomology. By fixing the deformation parameters mentioned before we can obtain the following: Firstly, the Lagrangian of the twisted theory is the free fermion resolution [4] of the $\operatorname{GL}(N \mid N)$ WZNW model without the boson-fermion interaction term. Secondly, the BRST current of the topological theory is one of the fermionic affine supercurrents of the model. Likewise the preimages of the BRST current and stress-energy tensor in the cohomology can be expressed in terms of the supercurrents. This in turn means that the full supergroup GL $(N \mid N)$ WZNW model including the boson-fermion interaction term by twisting is related to a supersymmetric deformation of the $\mathcal{N}=(2,2)$ world-sheet supersymmetric $\mathrm{GL}(N) \times \mathrm{GL}(N)$ WZNW model. We will see that the boson-fermion interaction terms correspond to an F-term deformation with a chiral field. The principal chiral field deformation is a D-term type of deformation, but it turns out that it is exact in the BRST-charge.

We also consider the important example of string theory on $\mathrm{AdS}_{3} \times \mathrm{S}^{3} \times \mathrm{T}^{4}$. We show that our construction is a novel choice of $\mathcal{N}=(2,2)$ world-sheet superalgebra, which only slightly differs from the standard choice in the supercurrent $G^{+}$that becomes the BRSTcurrent after twisting. The other supercurrent $G^{-}$agrees with standard string theory. With the new choice of superalgebra the string theory is related to the $\mathrm{U}(1,1 \mid 2) \times \mathrm{U}(1 \mid 1) \times \mathrm{U}(1 \mid 1)$ supergroup WZNW model. We also show that all interaction terms in the WZNW model are exact in the supercharge $G^{-}$that fitted with standard string theory. Finally, for the case with boundary we conjecture a solution to the Warner problem.

The article is organized as follows: In section 2 we will introduce the models and concepts that we need, this includes an introduction to both world-sheet supersymmetric models and supergroup WZNW models. Section 3 shows in detail how to get the relation between the world-sheet supersymmetric $\operatorname{GL}(N) \times \mathrm{GL}(N)$ WZNW model and the supergroup GL $(N \mid N)$ WZNW model. Here we also consider the supersymmetric deformations of the $\mathrm{GL}(N) \times \mathrm{GL}(N)$ WZNW model that corresponds to the boson-fermion interaction terms and the principal chiral field deformations. String theory on $\mathrm{AdS}_{3} \times \mathrm{S}^{3} \times \mathrm{T}^{4}$ is considered in section 4 along with the Warner problem for the case with boundary. We conclude with an outlook in section 5 .

\section{Superconformal and supergroup WZNW models}

In this section we introduce the two types of models that we study. Firstly, we review the construction of $\mathcal{N}=(2,2)$ superconformal symmetry in world-sheet supersymmetric 
WZNW models and, secondly, review Lie supergroup WZNW models and their free fermion resolution. We also briefly recall the construction of topological theories by twisting.

\subsection{Supersymmetric WZNW models}

We consider a world-sheet supersymmetric WZNW model whose target space is a Lie group, $G$. To define the model let us use a superspace notation where the world-sheet is a $2 \mid 2$ dimensional supersurface $\Sigma$ parameterized by complex coordinates $z, \bar{z}$ and two odd coordinates $\theta, \bar{\theta}$. The basic field, $\Gamma$, is then a map from this super world-sheet into the Lie group. It turns out that at the WZNW point, this field has a nice parametrization [17]. Let $\mathfrak{g}$ be the Lie algebra of $G$ and $\left\{t^{a}\right\}$ a basis. Then we define the fermionic Lie algebra valued fields

$$
\chi(z, \bar{z})=\chi_{a}(z, \bar{z}) t^{a} \quad \text { and } \quad \bar{\chi}(z, \bar{z})=\bar{\chi}_{a}(z, \bar{z}) t^{a} .
$$

Exponentiating these fields with an odd parameter gives Lie group valued fields. Furthermore, let $g$ be a bosonic Lie group valued field then we can parameterize the superfield $\Gamma$ as

$$
\Gamma=\exp (i \theta \chi) g \exp (-i \bar{\theta} \bar{\chi}) .
$$

To define the action of the WZNW model we need to fix some non-degenerate invariant bilinear form $(\cdot, \cdot)$ on the Lie algebra $\mathfrak{g}$ of $G$. The level $k$ of the model is here absorbed into the definition of the bilinear form $(\cdot, \cdot)$. The action of the world-sheet supersymmetric model is given by the standard WZNW action with $g$ replaced by $\Gamma$, and integration is over the super world-sheet $\Sigma$. In our parametrization it can be shown to have the form

$$
S_{\mathrm{WZNW}}^{\mathcal{N}=1}[\Gamma]=S_{\mathrm{WZNW}}^{(\mathrm{ren})}[g]+\frac{1}{2 \pi} \int d^{2} z(\chi, \bar{\partial} \chi)+(\bar{\chi}, \partial \bar{\chi}) .
$$

Here the bosonic part of the WZNW action has been renormalized by the Killing form $(\cdot, \cdot)_{\text {Kil. }}$

$$
(\cdot, \cdot)_{\text {ren }}=(\cdot, \cdot)+\frac{1}{2}(\cdot, \cdot)_{\text {Kil. }}
$$

We see that the fermions have been decoupled from the bosonic WZNW model.

The WZNW model $(2.3)$ has an $\mathcal{N}=(1,1)$ superconformal symmetry by construction. Below we will see that in some cases this can be enhanced to an $\mathcal{N}=(2,2)$ superconformal algebra.

\subsection{A Sugawara-like construction of the superconformal algebra}

This construction has been introduced in [16]. In this section, we construct the $\mathcal{N}=2$ superconformal algebra of the world-sheet super symmetric WZNW models. We restrict to the holomorphic sector, the anti-holomorphic currents are analogous.

The $\mathcal{N}=2$ superconformal currents consist of the chiral Virasoro field $T$, two fermionic fields $G^{ \pm}$with conformal weight $h_{G}=3 / 2$ and a bosonic $\mathrm{U}(1)$ current $U$ with weight one. The $\mathcal{N}=2$ superconformal algebra is encoded in the operator product expansions

$$
\begin{aligned}
G^{+}(z) G^{-}(w) & \sim \frac{c / 3}{(z-w)^{3}}+\frac{U(w)}{(z-w)^{2}}+\frac{\left(T+\frac{1}{2} \partial U\right)(w)}{(z-w)}, \\
U(z) G^{ \pm}(w) & \sim \frac{ \pm G^{ \pm}(w)}{(z-w)}, \quad U(z) U(w) \sim \frac{c / 3}{(z-w)^{2}} .
\end{aligned}
$$


Given an $\mathcal{N}=1$ superconformal WZNW model, as described in last subsection, there is a precise criterium whether it possesses an $\mathcal{N}=2$ superconformal symmetry. That is, let $\mathfrak{g}$ be the Lie algebra of the Lie group $G$. Suppose there exist two Lie subalgebras $\mathfrak{a}_{ \pm}$ such that

$$
\mathfrak{g}=\mathfrak{a}_{+} \oplus \mathfrak{a}_{-}
$$

Further, assume that $\mathfrak{a}_{ \pm}$are isotropic, i.e. the bi-linear form vanishes on them, then the world-sheet supersymmetric WZNW model possesses an $\mathcal{N}=2$ superconformal symmetry.

To explicitly construct the $\mathcal{N}=2$ currents we introduce some notation. Choose a basis $x_{i}$ of the Lie subalgebra $\mathfrak{a}_{+}$. With the help of our bi-linear form $(.,$.$) we can then fix a$ dual basis $x^{i}$ of $\mathfrak{a}_{-}$such that $\left(x_{i}, x^{j}\right)=\delta_{i}^{j}$. Our choice of basis implies that the Lie bracket takes the following form

$$
\begin{aligned}
{\left[x_{i}, x_{j}\right] } & =c_{i j}{ }^{k} x_{k}, \\
{\left[x^{i}, x^{j}\right] } & =f^{i j}{ }_{k} x^{k}, \\
{\left[x_{i}, x^{j}\right] } & =c_{k i}{ }^{j} x^{k}+f^{j k}{ }_{i} x_{k} .
\end{aligned}
$$

Here $c_{i j}{ }^{k}$ and $f^{i j}{ }_{k}$ are the structure constants of $\mathfrak{a}_{+}$and $\mathfrak{a}_{-}$, respectively. The last equation follows from the first two using the invariance of the bilinear form. For later convenience we define the element $\rho \in \mathfrak{g}$

$$
\rho:=-\left[x^{i}, x_{i}\right]=f^{i k}{ }_{i} x_{k}+c_{k i}{ }^{i} x^{k}=\rho^{k} x_{k}+\rho_{k} x^{k} .
$$

We denote the chiral affine currents corresponding to the generators $x_{i}$ and $x^{i}$ by $J_{i}(z)$ and $J^{i}(z)$. Using (2.7) and (2.4) their operator products are

$$
\begin{aligned}
J_{i}(z) J_{j}(w) & \sim \frac{\frac{1}{2}\left(x_{i}, x_{j}\right)_{\mathrm{Kil}} .}{(z-w)^{2}}+\frac{c_{i j}{ }^{k} J_{k}(w)}{(z-w)} \\
J_{i}(z) J^{j}(w) & \sim \frac{\delta_{i}{ }^{j}+\frac{1}{2}\left(x_{i}, x^{j}\right)_{\mathrm{Kil} .}}{(z-w)^{2}}+\frac{f^{j k}{ }_{i} J_{k}(w)+c_{k i}{ }^{j} J^{k}(w)}{(z-w)} \\
J^{i}(z) J^{j}(w) & \sim \frac{\frac{1}{2}\left(x^{i}, x^{j}\right)_{\mathrm{Kil}}}{(z-w)^{2}}+\frac{f^{i j}{ }_{k} J_{k}(w)}{(z-w)}
\end{aligned}
$$

The operator product expansions of the fields $\chi^{i}$ and $\chi_{i}$ take the form

$$
\begin{aligned}
\chi_{i}(z) \chi_{j}(w) & \sim 0, \\
\chi_{i}(z) \chi^{j}(w) & \sim \frac{\delta_{i}{ }^{j}}{(z-w)}, \\
\chi^{i}(z) \chi^{j}(w) & \sim 0 .
\end{aligned}
$$

All these fermions have conformal weight $h\left(\chi_{i}\right)=h\left(\chi^{i}\right)=1 / 2$.

We can now write the currents explicitly. The Virasoro tensor $T$ is in the standard Sugawara form

$$
T(z)=\frac{1}{2}\left(: J^{i} J_{i}:+: J_{i} J^{i}:+: \partial \chi^{i} \chi_{i}:-: \chi^{i} \partial \chi_{i}:\right)
$$


whereas the dimension $3 / 2$ fermionic currents are

$$
\begin{aligned}
& G^{+}(z)=J_{i} \chi^{i}-\frac{1}{2} c_{i j}{ }^{k}: \chi^{i} \chi^{j} \chi_{k}: \\
& G^{-}(z)=J^{i} \chi_{i}-\frac{1}{2} f^{i j}{ }_{k}: \chi_{i} \chi_{j} \chi^{k}: .
\end{aligned}
$$

Finally, the dimension one bosonic current $U$ is

$$
U(z)=: \chi^{i} \chi_{i}:+\rho^{k} J_{k}+\rho_{k} J^{k}+c_{m n}^{i} f_{j}^{m n}: \chi^{j} \chi_{i}: .
$$

Using the OPEs (2.9) and (2.10) one can verify that these currents satisfy the relations of the $\mathcal{N}=2$ superconformal algebra (2.5). The anti-chiral partners $\bar{T}, \bar{G}^{ \pm}$and $\bar{U}$ are constructed in complete analogy.

\subsection{Deformations}

The above construction can be slightly generalized [16]. Actually there exist a family of supersymmetric deformations defined by elements in $\mathfrak{a}_{\mathfrak{o}}$, the orthogonal complement of the direct sum of the derived subalgebras of $\mathfrak{a}_{+}$and $\mathfrak{a}_{-}$, i.e.

$$
\mathfrak{a}_{\mathfrak{o}}=\left\{x \in \mathfrak{g} \mid(x, y)=0 \forall y \in\left[\mathfrak{a}_{+}, \mathfrak{a}_{+}\right] \oplus\left[\mathfrak{a}_{-}, \mathfrak{a}_{-}\right]\right\} .
$$

Consider an element $\alpha=p^{i} x_{i}+q_{i} x^{i} \in \mathfrak{a}_{\mathfrak{o}}$. It follows from the definition of $\mathfrak{a}_{\mathfrak{o}}$ that the components $p^{i}$ and $q_{i}$ must satisfy

$$
c_{i j}{ }^{k} q_{k}=f^{i j}{ }_{k} p^{k}=0 .
$$

Given the element $\alpha$, the deformed currents of the $\mathcal{N}=2$ superconformal algebra are as follows

$$
\begin{aligned}
& U_{\alpha}(z)=U(z)+p^{i} I_{i}(z)-q_{i} I^{i}(z), \\
& T_{\alpha}(z)=T(z)+\frac{1}{2}\left(p^{i} \partial I_{i}(z)+q_{i} \partial I^{i}(z)\right) .
\end{aligned}
$$

Here we used the following set of level $k$ Lie superalgebra currents

$$
\begin{aligned}
& I_{i}=J_{i}-c_{i j}{ }^{k}: \chi^{j} \chi_{k}:-\frac{1}{2} f_{i}^{j k}: \chi_{j} \chi_{k}: \\
& I^{i}=J^{i}-f_{k}^{i j}: \chi_{j} \chi^{k}:-\frac{1}{2} c_{j k}{ }^{i}: \chi^{j} \chi^{k}: .
\end{aligned}
$$

The expressions for the deformed supercurrents $G^{ \pm}$are a bit simpler

$$
\begin{aligned}
G_{\alpha}^{+} & =G^{+}+q_{i} \partial \chi^{i}, \\
G_{\alpha}^{-} & =G^{-}+p^{i} \partial \chi_{i} .
\end{aligned}
$$

The deformation changes the central charge as

$$
c_{\alpha}=c-6 q_{i} p^{i} .
$$

This deformed $\mathcal{N}=2$ structure extends a deformation of the original $\mathcal{N}=1$ superconformal algebra, and will be important in our discussion. Note that the deformation simply changes the energy-momentum tensor by derivatives of the generalized currents $I_{i}, I^{i}$. In simple cases this is just adding background charges to the action. Indeed, as shown in [18] these deformations are closely related to spectral flow. 


\subsection{Topological conformal field theory}

It will be important for us that an $\mathcal{N}=2$ superconformal theory determine topological conformal theories by the twisting procedure. Here we follow [19].

In this paper we only need to consider the positive B-twist. Given an $\mathcal{N}=2$ superconformal theory as above, we define the energy-momentum tensor of the B-twisted theory by

$$
\begin{aligned}
& T_{\text {twisted }}^{+}(z)=T(z)+\frac{1}{2} \partial U(z), \\
& \bar{T}_{\text {twisted }}^{+}(\bar{z})=\bar{T}(\bar{z})+\frac{1}{2} \bar{\partial} \bar{U}(\bar{z}) .
\end{aligned}
$$

The twisted theory will by definition have central charge $c=0$. We see, as in the deformations above, that if we write $U=\partial \phi$ this will just add background charge to $\phi$ in the action, and again it can be seen as a spectral flow [20]. This means that the dimensions of the fields change and we now have the weights $h_{G^{+}}=1, h_{G^{-}}=2$ whereas $U$ still have weight one. The twisted theory is not in itself topological, but if we restrict ourselves to the states in the BRST-cohomology of $G^{+}$, we get a conformal topological theory. ${ }^{2}$ Indeed, from the $\mathcal{N}=2$ algebra (2.5) we see that the zero modes of $G^{+}+\bar{G}^{+}$satisfy

$$
\begin{aligned}
\left(G_{0}^{+}+\bar{G}_{0}^{+}\right)^{2} & =0, \\
{\left[G_{0}^{+}+\bar{G}_{0}^{+}, G^{-}(z)\right] } & =T_{\text {twisted }}^{+}(z), \\
{\left[G_{0}^{+}+\bar{G}_{0}^{+}, \bar{G}^{-}(\bar{z})\right] } & =\bar{T}_{\text {twisted }}^{+}(\bar{z}),
\end{aligned}
$$

which precisely is the algebra of a conformal topological theory with BRST charge $Q+\bar{Q} \equiv$ $G_{0}^{+}+\bar{G}_{0}^{+}$. The physical states are defined by the cohomology

$$
\mathcal{H}_{\text {phys }}=\frac{\operatorname{kernel}(Q+\bar{Q})}{\operatorname{image}(Q+\bar{Q})} .
$$

Note that $G^{-}$is the preimage of the twisted stress-energy tensor, and $U$ will be the preimage of the BRST charge $Q$ itself. Using these relations one can show that the physical correlation functions

$$
\left\langle\phi_{1}\left(z_{1}, \bar{z}_{1}\right) \ldots \phi_{n}\left(z_{n}, \bar{z}_{n}\right)\right\rangle_{\Sigma}
$$

will depend only on the fields $\phi_{i}$ and the topology of the world-sheet $\Sigma$, but not on the world-sheet positions $\left(z_{i}, \bar{z}_{i}\right)$. In the topological CFT the operator product expansion of physical fields takes the particularly simple form

$$
\phi_{i} \phi_{j} \sim c_{i j}{ }^{k} \phi_{k}
$$

\subsection{Supersymmetric deformations}

It is important to understand the moduli space of deformations preserving the superconformal algebra.

\footnotetext{
${ }^{2}$ Had we considered the negative B-twist with $T_{\text {twisted }}^{-}(z)=T(z)-\frac{1}{2} \partial U(z)$ the BRST operator would be $G^{-}$.
} 
Let us first relate our notation to the notation in [21] where the supercharges are denoted (in the Minkowski notation) $Q_{ \pm}$and $\bar{Q}_{ \pm}$where the index \pm denotes chirality and hence is related to our bar notation. On the other hand, the bar notation in [21] is related to hermitian conjugation of the supercharges. In our case this corresponds to the \pm superscript. We have

$$
\left\{G_{-1 / 2}^{+}, G_{-1 / 2}^{-}\right\}=2 L_{-1}, \quad\left\{\bar{G}_{-1 / 2}^{+}, \bar{G}_{-1 / 2}^{-}\right\}=2 \bar{L}_{-1}
$$

whereas in [21] the non-zero anti-commutators are (in Minkowski space and with zero central charges for the supersymmetry algebra)

$$
\left\{Q_{ \pm}, \bar{Q}_{ \pm}\right\}=H \pm P=-2 i \partial / \partial x^{ \pm} .
$$

Taking $z=x^{2}+i x^{1}$ and Wick rotating as $x^{2}=i x^{0}$ we get $L_{-1}=-\partial_{z}=i \partial / \partial x^{+}$and $\bar{L}_{-1}=-\partial_{\bar{z}}=i \partial / \partial x^{-}$. So we choose the identification

$$
\begin{array}{ll}
Q_{+}=i G_{-1 / 2}^{-}, & \bar{Q}_{+}=i G_{-1 / 2}^{+}, \\
Q_{-}=i \bar{G}_{-1 / 2}^{-}, & \bar{Q}_{-}=i \bar{G}_{-1 / 2}^{-} .
\end{array}
$$

In the superfield formalism we introduce covariant superderivatives $D_{ \pm}$and $\bar{D}_{ \pm}$. A chiral field, $\Phi_{++}$, is a superfield with $\bar{D}_{ \pm} \Phi_{++}=0$. Correspondingly an anti-chiral field has $D_{ \pm} \Phi_{--}=0$, a twisted chiral field has $\bar{D}_{+} \Phi_{+-}=D_{-} \Phi_{+-}=0$, and finally a twisted anti-chiral field has $D_{+} \Phi_{-+}=\bar{D}_{-} \Phi_{-+}=0$. The component fields of a chiral superfield form a representation of the supersymmetry algebra. Specifically we find that a field $\phi_{a b}$ is the lowest component of a (twisted) (anti-)chiral superfield $\Phi_{a b}$ if and only if

$$
\left[G_{-1 / 2}^{a}, \phi_{a b}\right]=\left[\bar{G}_{-1 / 2}^{b}, \phi_{a b}\right]=0 .
$$

i.e. if it belongs to the (ab)-chiral ring (i.e. $(++)$ is $(\mathrm{cc})$ etc. in standard notation). The middle components are then given by

$$
\psi_{a b}=-\left[G_{-1 / 2}^{-a}, \phi_{a b}\right], \quad \bar{\psi}_{a b}=-\left[\bar{G}_{-1 / 2}^{-b}, \phi_{a b}\right],
$$

and the highest order F-term component is

$$
F_{a b}=-\left\{G_{-1 / 2}^{-a},\left[\bar{G}_{-1 / 2}^{-b}, \phi_{a b}\right]\right\} .
$$

A functional of a chiral field is again a chiral field which is reflected by the ring nature of the chiral ring. We can also take world-sheet derivatives and preserve chirality.

Using these fields we can build actions that, at least classically, are invariant under the supersymmetry transformations. We have two type of terms: F-terms which only depend on one type of fields and which always will have the form (2.30). For F-terms the lowest component field $\phi_{a b}$ needs to have dimension $(1 / 2,1 / 2)$. The second type of terms are D-terms which consist of different types of fields and can change the metric or B-field. These terms needs to have dimension zero.

By (2.24) we see that under a positive B-twist, the F-term perturbations generated by anti-chiral, twisted chiral or twisted anti-chiral superfields are all exact up to total derivatives. That is, they can be written as $\left[G_{-1 / 2}^{+}+\bar{G}_{-1 / 2}^{+}, \phi\right]+$ total derivatives, for some field $\phi$. 


\subsection{Lie supergroup WZNW models}

Using the twist procedure from last subsection we want to relate world-sheet supersymmetric theories to WZNW models of type I Lie supergroups. In this section we recall results on these from [4].

We consider a type I Lie superalgebra $\mathfrak{g}$. Examples of fundamental matrix representations are as follows:

Example 2.1. $g l(n \mid m)$ is given by

$$
g l(n \mid m)=\left\{\left(\begin{array}{c|c}
A \mid B \\
\hline C \mid D
\end{array}\right)\right\},
$$

where the bosonic matrices $A$ and $D$ are square matrices of size $n \times n$ and $m \times m$, and the odd matrices $B$ and $C$ respectively are of size $n \times m$ and $m \times n$. The supertrace is a supersymmetric non-degenerate invariant bilinear form and it is defined via

$$
\operatorname{str}\left(\begin{array}{c|c}
A & B \\
\hline C & D
\end{array}\right)=\operatorname{tr} A-\operatorname{tr} D .
$$

Example 2.2. $\operatorname{sl}(n \mid m)$

$$
s l(n \mid m)=\{X \in g l(n \mid m) \mid \operatorname{str} X=0\},
$$

for $n \neq m$. If $n=m \operatorname{sl}(n \mid n)$ is not simple, in this case one obtains the projective unitary superalgebra $\operatorname{psl}(n \mid n)$ as the quotient of $\operatorname{sl}(n \mid n)$ by its one dimensional ideal $\mathcal{I}$ generated by the identity matrix $1_{2 n}$, i.e. $\operatorname{psl}(n \mid n)=\operatorname{sl}(n \mid n) / \mathcal{I}$.

Following [4] we denote the upper fermionic generators, the positive fermionic roots, by $S_{1}^{a}$, the lower fermionic by $S_{2 a}$ and the bosonic by $K^{i}$. As a non-degenerate invariant bilinear form, we use the supertrace

$$
\langle A, B\rangle=k \operatorname{str}(A B) .
$$

The bosonic part of this metric is denoted $\kappa^{i j}$, and the fermionic part is

$$
\left\langle S_{1}^{a}, S_{2 b}\right\rangle=k \delta_{b}^{a} .
$$

We parameterize a supergroup valued field as

$$
g=e^{c} g_{B} e^{\bar{c}},
$$

where we have introduced fermionic fields $c$ and $\bar{c}$

$$
c=c^{a} S_{2 a}, \quad \bar{c}=\bar{c}_{a} S_{1}^{a} .
$$

The fermions transform in some representation of the bosonic algebra. We introduce the representation matrices $R^{i}$ by

$$
\left[K^{i}, S_{1}^{a}\right]=-\left(R^{i}\right)^{a}{ }_{b} S_{1}^{b},
$$


which implies

$$
\begin{aligned}
{\left[K^{i}, S_{2 a}\right] } & =S_{2 b}\left(R^{i}\right)^{b}{ }_{a}, \\
{\left[S_{1}^{a}, S_{2 b}\right] } & =-k\left(R^{i}\right)^{a}{ }_{b} \kappa_{i j} K^{j} .
\end{aligned}
$$

By $R\left(g_{B}\right)$ we denote the representation of the group element $g_{B}$.

A first order formalism for the fermions (called the free fermion resolution [4]) is obtained by introducing auxiliary dimension $1 b$-ghosts to match the fermionic fields $c$ :

$$
b=b_{a} S_{1}^{a}, \quad \bar{b}=\bar{b}^{a} S_{2 a} .
$$

The WZNW action then becomes

$$
\begin{aligned}
S^{\mathrm{WZNW}}[g] & =S_{0}+S_{\text {pert }} \\
S_{0} & =S\left[g_{B}\right]_{\mathrm{ren}+\text { dil }}+\frac{1}{2 \pi} \int_{\Sigma} d \tau d \sigma \operatorname{str}(b \bar{\partial} c)-\operatorname{str}(\bar{b} \partial \bar{c}) \\
S_{\text {pert }} & =-\frac{1}{4 \pi k} \int_{\Sigma} d \tau d \sigma \operatorname{str}\left(\operatorname{Ad}\left(g_{B}\right)(\bar{b}) b\right),
\end{aligned}
$$

where $S\left[g_{B}\right]_{\text {ren+dil }}$ is a renormalized version of the bosonic WZNW action plus dilatonic terms. Written out in components we get

$$
\begin{aligned}
S^{\mathrm{WZNW}}[g]=S_{r e n}^{\mathrm{WZNW}}\left[g_{B}\right]-\frac{1}{8 \pi} \int d z^{2} \sqrt{h} \mathcal{R}^{(2)} \ln \operatorname{det} R\left(g_{B}\right) \\
+\frac{1}{2 \pi} \int d z^{2}\left(b_{a} \bar{\partial} c^{a}-\bar{b}_{a} \partial \bar{c}^{a}-b_{a} R\left(g_{B}\right)^{a}{ }_{b} \bar{b}^{b}\right),
\end{aligned}
$$

The extra terms are due to the change in the quantum measure. This gives rise to the Fradkin-Tseytlin term where $h$ is the determinant of the world-sheet metric and $\mathcal{R}^{(2)}$ is the world-sheet curvature. Further, there is a renormalization of the metric in the bosonic part given by

$$
\left\langle K_{B}^{i}, K_{B}^{j}\right\rangle_{r e n}=\kappa^{i j}-\gamma^{i j}, \quad \gamma^{i j}=\operatorname{tr} R^{i} R^{j} .
$$

Here we have denoted the bosonic currents corresponding to this renormalized metric by $K_{B}^{i}$. Even for simple superalgebras $\mathfrak{g}, \gamma$ may not be proportional to $\kappa$.

The affine currents now take the following form (factors of $k$ are absorbed in the metric)

$$
J=-\partial g g^{-1}=-\partial c+K_{B}+\left[c, K_{B}\right]+\frac{1}{k} b+\frac{1}{k}:[c, b]:+\frac{1}{2 k}:[c,:[c, b]:]: .
$$

We have to be careful with signs when writing the currents in components [18]. In our case of type I superalgebras we have

$$
J^{a}(z)=\left\{\begin{array}{cc}
\left(J(z), t^{a}\right) & \text { if } t^{a} \text { in } \mathfrak{g}_{B} \oplus \mathfrak{g}_{+} \\
-\left(J(z), t^{a}\right) & \text { if } t^{a} \text { in } \mathfrak{g}_{-}
\end{array}\right.
$$


This gives us (with $\left.K_{B}(z)=K_{B}^{i}(z) \kappa_{i j} K^{j}\right)$

$$
\begin{aligned}
J^{K^{i}}(z) & =J^{K_{B}^{i}}(z)+b_{a}(z)\left(R^{i}\right)^{a}{ }_{b} c^{b}(z), \\
J^{S_{1}^{a}}(z) & =k \partial c^{a}(z)+k\left(R^{i}\right)^{a}{ }_{b}{ }^{\kappa_{i j}} c^{b}(z) J^{K_{B}^{j}}(z)-\frac{k}{2}\left(R^{i}\right)^{a}{ }_{b} \kappa_{i j}\left(R^{j}\right)^{c}{ }_{d} b_{c} c^{b} c^{d}(z), \\
J^{S_{2 a}}(z) & =-b_{a}(z)
\end{aligned}
$$

where right-nested normal ordering is understood. These currents satisfy the OPEs

$$
J^{a}(z) J^{b}(w) \sim \frac{k \operatorname{str}\left(t^{a} t^{b}\right)}{(z-w)^{2}}+\frac{f_{c}^{a b} J^{c}}{z-w},
$$

where $f^{a b}{ }_{c}$ are the structure constants. This can be checked using the OPEs for $K_{B}^{i}$ remembering the renormalization (2.43), and the OPEs for the fermions

$$
b_{a}(z) c^{a}(w) \sim \frac{1}{z-w} .
$$

Finally, the energy-momentum tensor can be written as

$$
T^{\mathrm{FF}}=\frac{1}{2}\left(J^{K_{B}^{i}} \Omega_{i j} J^{K_{B}^{j}}+\operatorname{tr}\left(\Omega R^{i}\right) \kappa_{i j} \partial J^{K_{B}^{j}}\right)-b_{a} \partial c^{a},
$$

where the full-renormalized metric $\Omega$ has the bosonic and fermionic parts:

$$
\begin{aligned}
& \left(\Omega^{-1}\right)^{i j}=\kappa^{i j}-\gamma^{i j}+\frac{1}{2} f_{n}^{i m} f_{m}^{j n}, \\
& \left(\Omega^{-1}\right)_{b}^{a}=\delta_{b}^{a}+\left(R^{i} \kappa_{i j} R^{j}\right)_{b}^{a} .
\end{aligned}
$$

Note, that the bosonic part of the energy-momentum tensor is a deformation of the Sugawara Virasoro field. This resembles very much the form of the $\mathcal{N}=2$ deformations considered in the last section (2.16) and the twisting (2.19). In the following we will explain the relation.

\section{From world-sheet supersymmetry to supergroups}

In this section we will understand a relation between world-sheet supersymmetric WZNW models and Lie supergroup WZNW models. We start with a $\mathcal{N}=(2,2)$ superconformal $\mathrm{GL}(\mathrm{N}) \times \mathrm{GL}(\mathrm{N})$ WZNW model. We find a truly marginal operator $\Phi$, i.e. a perturbation that preserves the superconformal algebra. This operator couples bosonic fields with the world-sheet fermions. Then we perform a topological B-twist. This twist is identified with the $\mathrm{GL}(\mathrm{N} \mid \mathrm{N})$ WZNW model in the form sketched as

$$
\begin{aligned}
& S_{\mathrm{GL}(\mathrm{N}) \times \mathrm{GL}(\mathrm{N})}^{\mathcal{N}=(2,2)}+\frac{1}{2 \pi} \int d \tau d \sigma \Phi \stackrel{\text { B-twist }}{\longrightarrow} S_{\mathrm{GL}(\mathrm{N} \mid \mathrm{N})} \\
& G^{+}(z) \stackrel{\text { B-twist }}{\longrightarrow} J^{F}(z) \\
& G^{-}(z) \stackrel{\text { B-twist }}{\longrightarrow} \sum J^{F}(z) J^{B}(z)+\partial J^{F}(z) \\
& U(z) \stackrel{\text { B-twist }}{\longrightarrow} J^{B}(z) \text {. }
\end{aligned}
$$


I.e. the twisted action is the supergroup WZNW model action, and the twisted super currents can be identified with affine Lie super algebra currents. Here $J^{F}$ and $J^{B}$ denote fermionic and bosonic currents in $\mathrm{GL}(\mathrm{N} \mid \mathrm{N})$. The goal now is to make the above sketch (3.1) precise.

We start by introducing the GL(N|N) WZNW model, then we consider the $\mathcal{N}=(2,2)$ $\mathrm{GL}(\mathrm{N}) \times \mathrm{GL}(\mathrm{N})$ WZNW model, perform the B-twist and relate this to the $\mathrm{GL}(\mathrm{N} \mid \mathrm{N})$ WZNW model. We introduce the boson-fermion interaction term $\Phi$ and explain its exactly marginality. Finally, we consider perturbations by the principal chiral field and show that it is a D-term.

\subsection{Some properties of the GL(N|N) WZNW model}

In this section, we extend section 2.6 in the special cases of the Lie supergroups GL $(\mathrm{N} \mid \mathrm{N})$. A convenient basis for the Lie superalgebra $\operatorname{gl}(\mathrm{N} \mid \mathrm{N})$ is $\left\{E_{\epsilon}^{\alpha \beta}, F_{\epsilon}^{\alpha \beta} \mid 1 \leq \alpha, \beta \leq N, \epsilon= \pm\right\}$, where the generators $E$ are bosonic and $F$ are fermionic. Compared to section 2.6 the bosonic indices $i, j, \ldots$ are now each replaced by a triplet $\left(\begin{array}{c}\alpha \beta \\ \epsilon\end{array}\right)$ and the fermionic indices $a, b, \ldots$ are each replaced by a doublet $(\alpha \beta)$. The advantage of this notation is that the invariant bilinear form and the super commutation relations are easy to express. The metric (2.34) takes the form

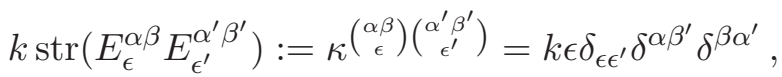

$$
\begin{aligned}
& k \operatorname{str}\left(F_{\epsilon}^{\alpha \beta} F_{\epsilon^{\prime}}^{\alpha^{\prime} \beta^{\prime}}\right)=k \varepsilon_{\epsilon \epsilon^{\prime}} \delta^{\alpha \beta^{\prime}} \delta^{\beta \alpha^{\prime}},
\end{aligned}
$$

where $\varepsilon_{\epsilon \epsilon^{\prime}}$ is the antisymmetric symbol with $\epsilon_{+-}=1$.

The non-vanishing Lie super algebra relations are

$$
\begin{aligned}
{\left[E_{\epsilon}^{\alpha \beta}, E_{\epsilon^{\prime}}^{\gamma \delta}\right] } & =\delta_{\epsilon, \epsilon^{\prime}}\left(\delta^{\beta \gamma} E_{\epsilon}^{\alpha \delta}-\delta^{\alpha \delta} E_{\epsilon}^{\gamma \beta}\right), \\
{\left[E_{\epsilon}^{\alpha \beta}, F_{\epsilon^{\prime}}^{\gamma \delta}\right] } & =\delta_{\epsilon, \epsilon^{\prime}} \delta^{\beta \gamma} F_{\epsilon}^{\alpha \delta}-\delta_{-\epsilon, \epsilon^{\prime}} \delta^{\alpha \delta} F_{\epsilon^{\prime}}^{\gamma \beta}, \\
\left\{F_{\epsilon}^{\alpha \beta}, F_{\epsilon^{\prime}}^{\gamma \delta}\right\} & =\delta_{-\epsilon, \epsilon^{\prime}}\left(\delta^{\beta \gamma} E_{\epsilon}^{\alpha \delta}+\delta^{\alpha \delta} E_{\epsilon^{\prime}}^{\gamma \beta}\right) .
\end{aligned}
$$

Following (2.37) we define the fermionic fields

$$
\begin{array}{lll}
c=c^{\beta \alpha} F_{-}^{\alpha \beta}, & \bar{c}=\bar{c}^{\alpha \beta} F_{+}^{\alpha \beta}, \\
b=b_{\alpha \beta} F_{+}^{\alpha \beta}, & \bar{b}=\bar{b}_{\beta \alpha} F_{-}^{\alpha \beta} .
\end{array}
$$

These satisfy the OPEs

$$
c^{\alpha \beta}(z) b_{\gamma \delta}(w) \sim \frac{\delta_{\alpha \gamma} \delta_{\beta \delta}}{(z-w)},
$$

and correspondingly for the bared fields.

The boson-fermion super commutation relation in eq. (3.4) determines the representation matrices $R$ by (2.38)

$$
\left(R^{\left(\begin{array}{c}
\alpha \beta \\
\epsilon
\end{array}\right)}\right)_{\gamma^{\prime} \delta^{\prime}}^{\gamma \delta}=-\delta_{\epsilon,+} \delta^{\beta \gamma} \delta_{\gamma^{\prime}}^{\alpha} \delta_{\delta^{\prime}}^{\delta}+\delta_{\epsilon,-} \delta^{\alpha \delta} \delta_{\gamma^{\prime}}^{\gamma} \delta_{\delta^{\prime}}^{\beta} .
$$


This gives the correction to the bosonic metric from decoupling the fermions (2.43)

$$
\gamma^{\left(\begin{array}{c}
\alpha \beta \\
\epsilon
\end{array}\right)\left(\begin{array}{c}
\gamma \delta \\
\epsilon^{\prime}
\end{array}\right)}=\delta_{\epsilon, \epsilon^{\prime}} N \delta^{\alpha \delta} \delta^{\beta \gamma}-\delta_{-\epsilon, \epsilon^{\prime}} \delta^{\alpha \beta} \delta^{\gamma \delta},
$$

and the bosonic Killing metric $f_{n}^{i m} f_{m}^{j n}$ is

$$
\kappa_{\text {Killing }}^{\left(\begin{array}{c}
\alpha \beta \\
\epsilon
\end{array}\right)\left(\begin{array}{c}
\gamma \delta \\
\epsilon^{\prime}
\end{array}\right)}=2 \delta_{\epsilon, \epsilon^{\prime}}\left(N \delta^{\alpha \delta} \delta^{\beta \gamma}-\delta^{\alpha \beta} \delta^{\gamma \delta}\right)
$$

The GL $(\mathrm{N} \mid \mathrm{N})$ currents (2.46) are then given by

$$
\begin{aligned}
& J^{E_{\epsilon}^{\alpha \beta}}=J_{B}^{E_{\epsilon}^{\alpha \beta}}-\delta_{\epsilon,+} b_{\beta \gamma} c^{\alpha \gamma}+\delta_{\epsilon,-} b_{\gamma \alpha} c^{\gamma \beta}, \\
& J^{F_{-}^{\alpha \beta}}=-b_{\beta \alpha}, \\
& J^{F_{+}^{\alpha \beta}}=k \partial c^{\alpha \beta}-c^{\gamma \beta} J_{B}^{E_{+}^{\alpha \gamma}}-c^{\alpha \gamma} J_{B}^{E_{-}^{\gamma \beta}}-b_{\gamma \delta} c^{\gamma \beta} c^{\alpha \delta} .
\end{aligned}
$$

$J_{B}$ denote the bosonic currents with the renormalized metric (2.43).

Similarly the anti-holomorphic currents are

$$
\begin{aligned}
\bar{J}^{E_{\epsilon}^{\alpha \beta}} & =\bar{J}_{B}^{E_{\epsilon}^{\alpha \beta}}+\delta_{\epsilon,+} \bar{b}_{\alpha \gamma} \bar{c}^{\beta \gamma}-\delta_{\epsilon,-} b_{\gamma \beta} \bar{c}^{\gamma \alpha}, \\
\bar{J}_{-}^{F_{-}^{\alpha \beta}} & =k \partial \bar{c}^{\beta \alpha}+\bar{c}^{\gamma \alpha} \bar{J}_{B}^{E_{+}^{\gamma \beta}}+\bar{c}^{\beta \gamma} \bar{J}_{B}^{E_{-}^{\alpha \gamma}}-\bar{b}_{\gamma \delta} \bar{c}^{\gamma \alpha} \bar{c}^{\beta \delta} \\
\bar{J}_{+}^{\alpha \beta} & =\bar{b}_{\alpha \beta} .
\end{aligned}
$$

We also need the energy-momentum tensor. The bosonic and fermionic fullyrenormalized metrics (2.50) are given by

$$
\begin{aligned}
\left(\Omega^{-1}\right)^{\left(\begin{array}{c}
\alpha \beta \\
\epsilon
\end{array}\right)\left(\begin{array}{c}
\gamma \delta \\
\epsilon^{\prime}
\end{array}\right)}=k \epsilon \delta_{\epsilon \epsilon^{\prime}} \delta^{\alpha \delta} \delta^{\beta \gamma}-\epsilon \epsilon^{\prime} \delta^{\alpha \beta} \delta^{\gamma \delta}, \\
\left(\Omega^{-1}\right)_{(\gamma \delta)}^{(\alpha \beta)}=\delta_{\gamma}^{\alpha} \delta_{\delta}^{\beta} .
\end{aligned}
$$

The holomorphic component of the stress tensor is then

$$
T^{\mathrm{FF}}=\frac{1}{2} J_{B}^{E_{\epsilon}^{\alpha \beta}} \Omega_{\left(\begin{array}{c}
\alpha \beta \\
\epsilon
\end{array}\right)\left(\begin{array}{c}
\gamma \delta \\
\epsilon^{\prime}
\end{array}\right)} J_{B}^{E_{\epsilon^{\prime}}^{\gamma \delta}}-\frac{N}{2 k} \sum_{\alpha}\left(\partial J_{B}^{E_{+}^{\alpha \alpha}}+\partial J_{B}^{E_{-}^{\alpha \alpha}}\right)-b_{\alpha \beta} \partial c^{\alpha \beta} .
$$

\subsection{The $\mathcal{N}=(2,2) \mathbf{G L}(\mathbf{N}) \times \mathbf{G L}(\mathbf{N})$ WZNW model}

We now consider the world-sheet supersymmetric GL(N) $\times$ GL $(\mathrm{N})$ WZNW model that is related to the GL(N|N) WZNW model.

We denote the generators of $\mathfrak{g}=\operatorname{gl}(\mathrm{N}) \oplus \operatorname{gl}(\mathrm{N})$ by $E_{\sigma}^{\alpha \beta}, \sigma= \pm$, with relations

$$
\left[E_{\epsilon}^{\alpha \beta}, E_{\epsilon^{\prime}}^{\gamma \delta}\right]=\delta_{\epsilon, \epsilon^{\prime}}\left(\delta^{\beta \gamma} E_{\epsilon}^{\alpha \delta}-\delta^{\alpha \delta} E_{\epsilon}^{\gamma \beta}\right) .
$$

Furthermore we start with a metric given by

$$
\kappa_{\text {start }}\left(E_{\epsilon}^{\alpha \beta}, E_{\epsilon^{\prime}}^{\gamma \delta}\right)=\kappa_{\text {start }}^{\left(\begin{array}{c}
\alpha \beta \\
\epsilon
\end{array}\right)\left(\begin{array}{c}
\gamma \delta \\
\epsilon^{\prime}
\end{array}\right)}=k \epsilon \delta_{\epsilon \epsilon^{\prime}} \delta^{\alpha \delta} \delta^{\beta \gamma}-\epsilon \epsilon^{\prime} \delta^{\alpha \beta} \delta^{\gamma \delta} .
$$

This is the same as the fully-renormalized metric in equation (3.13). Note that this metric only differs from the standard trace metric in the U(1) parts. Thus it really only implies 
a simple field redefinition in the $\mathrm{U}(1)$ fields. The metric is chosen so that after decoupling the fermions, we get the metric of the free fermion resolution (2.43).

Define the Manin triple $\left(\mathfrak{g}, \mathfrak{a}_{+}, \mathfrak{a}_{-}\right)$corresponding to our starting metric as

$$
\begin{aligned}
& \mathfrak{g}=\mathfrak{a}_{+} \oplus \mathfrak{a}_{-} \\
& \mathfrak{a}_{+}=\operatorname{span}\left\{x_{\alpha \beta} \mid x_{\alpha \beta}=E_{+}^{\alpha \beta}+E_{-}^{\alpha \beta}\right\} \\
& \mathfrak{a}_{-}=\operatorname{span}\left\{x^{\alpha \beta} \mid x^{\alpha \alpha}=\frac{E_{+}^{\alpha \alpha}-E_{-}^{\alpha \alpha}}{2 k}+\frac{1}{2 k^{2}} \operatorname{Id}, x^{\beta \alpha}=\frac{E_{+}^{\alpha \beta}}{k}\right. \\
&\text { and } \left.x^{\alpha \beta}=-\frac{E_{-}^{\beta \alpha}}{k} \text { for } \alpha>\beta\right\} .
\end{aligned}
$$

where $\mathrm{Id}=\mathrm{Id}_{+}+\mathrm{Id}_{-}$denotes the central element given by the sum of the two $\mathrm{u}(1)$ generators $\operatorname{Id}_{\epsilon}=\sum_{\alpha} E_{\epsilon}^{\alpha \alpha}$. Note that $\mathfrak{a}_{+}$forms the Lie algebra $\mathrm{gl}(\mathrm{N})$ and $\mathfrak{a}_{-}$is a solvable Lie subalgebra of $\mathfrak{g}$ and both are isotropic. Hence we have a Manin triple. As a basis for $\mathfrak{a}_{+}$and $\mathfrak{a}_{-}$we use the $x_{\alpha \beta}$ and $x^{\alpha \beta}$ introduced in (3.17). Recall that constructions of the $\mathcal{N}=2$ superconformal algebra are parameterized by the orthogonal complement of the direct sum of the derived subalgebras of $\mathfrak{a}_{ \pm}$. This is

$$
\mathfrak{a}_{\mathfrak{o}}=\operatorname{span}\left\{x_{\alpha \alpha}, \sum_{\alpha} x^{\alpha \alpha}\right\} .
$$

For us the choice of $\mathcal{N}=2$ superconformal algebra given by $\gamma$ in $\mathfrak{a}_{\mathfrak{o}}$

$$
\gamma=-k \sum_{\alpha} x^{\alpha \alpha}-\sum_{\alpha} \frac{1}{2 k}(2 N-2 \alpha+1) x_{\alpha \alpha}
$$

is important. It implies (2.18) that the central charge is $c_{\gamma}=0$. In order to display the superconformal structure following sections 2.2 and 2.3 we need two more ingredients. First, the structure constants of $\mathfrak{a}_{+} \cong \operatorname{gl}(\mathrm{N})$ are denoted $c_{a b}{ }^{c}$. Those of $\mathfrak{a}_{-}$we call $f^{a b}{ }_{c}$ and they can be extracted from (3.15). Second the element $\rho$ (see (2.8)) is

$$
\rho=\frac{1}{k} \sum_{\alpha}(N-2 \alpha+1) x_{\alpha \alpha} .
$$

Further, introduce the bosonic currents $J_{\alpha \beta}$ of $\mathfrak{a}_{+}, J^{\alpha \beta}$ of $\mathfrak{a}_{-}$, the and fermionic fields $\chi_{\alpha \beta}$, and their partners $\chi^{\alpha \beta}$ with OPEs

$$
\chi_{\alpha \beta}(z) \chi^{\gamma \delta}(w) \sim \frac{\delta_{\alpha}^{\gamma} \delta_{\beta}^{\delta}}{(z-w)} .
$$

The $\mathcal{N}=2$ superconformal algebra can now be written down using sections 2.2 and 2.3, we will display them in a moment after a performing the B-twist. The B-twist changes the Virasoro field by a linear dilaton term (2.19). This implies that in the twisted theory the fermions $\chi^{\alpha \beta}$ have conformal dimension zero while the $\chi_{\alpha \beta}$ have weight one. Thus we can identify the fermions $\chi$ with the $b c$ ghosts of the free fermion resolution. We choose to identify as

$$
c^{\alpha \beta}=-\chi^{\beta \alpha}, \quad b_{\alpha \beta}=-\chi_{\beta \alpha} .
$$


Moreover, we identify the bosonic currents with the bosonic currents from the free fermion resolution of $\mathrm{GL}(\mathrm{N} \mid \mathrm{N})$ which have the same OPEs due to our choice of metric (3.16). The two stress-energy tensors then match due to our choice of the deformation parameter $\gamma$ in (3.19). This means that the action of the twisted topological theory is the same as the free fermion resolution action without the boson-fermion interaction term, i.e. the action $S_{0}$ in $(2.41)$.

We can now show that the twisted world-sheet supersymmetric currents are expressed by affine superalgebra currents. First consider the deformed supersymmetry current $G_{\gamma}^{+}$ (which after twisting has conformal dimension one) given by (2.17)

$$
G_{\gamma}^{+}=J_{\alpha \beta} \chi^{\alpha \beta}-\frac{1}{2} c_{\alpha \beta, \delta \rho}{ }^{\sigma \lambda} \chi^{\alpha \beta} \chi^{\delta \rho} \chi_{\sigma \lambda}-k \sum_{\alpha} \partial \chi^{\alpha \alpha}
$$

Comparing with (3.11) we observe that $G_{\gamma}^{+}$is a fermionic gl $(\mathrm{N} \mid \mathrm{N})$ superalgebra current ${ }^{3}$

$$
G_{\gamma}^{+}=\sum_{\alpha} J_{+}^{F_{+}^{\alpha}}
$$

The $\mathrm{U}(1)$ current is by $(2.16)$ given by

$$
U_{\gamma}=\frac{1}{2 k} \sum_{\alpha}(-2 \alpha+1) J_{\alpha \alpha}+k \sum_{\alpha} J^{\alpha \alpha}+\chi^{\alpha \beta} \chi_{\alpha \beta}+\frac{1}{k} \sum_{\alpha, \beta}(\alpha-\beta) \chi^{\alpha \beta} \chi_{\alpha \beta} .
$$

Again comparison with (3.11) gives the following identification with a bosonic $\operatorname{gl}(N \mid N)$ superalgebra current

$$
U_{\gamma}=\frac{1}{2 k} \sum_{\alpha}(k+N+1-2 \alpha) J^{E_{+}^{\alpha \alpha}}+\frac{1}{2 k} \sum_{\alpha}(-k+N+1-2 \alpha) J^{E_{-}^{\alpha \alpha}} .
$$

Finally, the $G^{-}$current of conformal weight two which is given by $(2.17)$ can be written as

$$
\begin{aligned}
G_{\gamma}^{-}= & \frac{1}{2 k} \sum_{\alpha} J^{F_{-}^{\alpha \alpha}}\left(J^{E_{+}^{\alpha \alpha}}-J^{E_{-}^{\alpha \alpha}}+\frac{1}{k} \sum_{\beta}\left(J^{E_{+}^{\beta \beta}}+J^{E_{-}^{\beta \beta}}\right)\right)-\frac{1}{k} \sum_{\alpha>\beta} J_{-}^{F_{-}^{\alpha \beta}} J^{E_{-}^{\beta \alpha}} \\
& +\frac{1}{k} \sum_{\alpha<\beta} J^{F_{-}^{\alpha \beta}} J^{E_{+}^{\beta \alpha}}-\frac{1}{2 k} \sum_{\alpha}(2 N-2 \alpha+1) \partial J^{F_{-}^{\alpha \alpha}} .
\end{aligned}
$$

Note that this expression is normal ordered which is important for the coefficients of the derivative terms.

So far we only considered the holomorphic currents. Let us now state the analogous results for the right-moving part. We choose to use the same Manin decomposition. The deformation parameter $\bar{\gamma}$ is slightly different,

$$
\bar{\gamma}=\sum_{\alpha}\left(-\frac{1}{2 k}(-2 \alpha+1) x_{\alpha \alpha}+k x^{\alpha \alpha}\right) .
$$

\footnotetext{
${ }^{3}$ To get the comparison of the stress-energy tensor of the $\operatorname{GL}(N) \times \operatorname{GL}(N)$ and $\operatorname{GL}(N \mid N)$ models we had to fix all the parameters in $\gamma(3.19)$ except one. Getting this relation fixes the last parameter.
} 
We now perform again the +-twist on the right-moving currents and identify the superconformal currents with affine superalgebra currents. First, the energy-momentum tensors match. The identification of the ghost system in this case is

$$
\bar{c}^{\alpha \beta}=\bar{\chi}^{\alpha \beta}, \quad \bar{b}_{\alpha \beta}=\bar{\chi}_{\alpha \beta},
$$

to get a matching between the supersymmetric currents and the super affine currents.

Identification of the currents are now

$$
\begin{aligned}
\bar{G}_{\bar{\gamma}}^{+}= & \sum_{\alpha} \bar{J}_{-}^{F_{-}^{\alpha \alpha}} \\
\bar{U}_{\bar{\gamma}}= & \frac{1}{2 k} \sum_{\alpha}(-k+N+1-2 \alpha) \bar{J}^{E_{+}^{\alpha \alpha}}+\frac{1}{2 k} \sum_{\alpha}(k+N+1-2 \alpha) \bar{J}^{E_{-}^{\alpha \alpha}} \\
\bar{G}_{\bar{\gamma}}^{-}= & \frac{1}{2 k} \sum_{\alpha} \bar{J}_{+}^{F_{+}^{\alpha \alpha}}\left(\bar{J}^{E_{+}^{\alpha \alpha}}-\bar{J}^{E_{-}^{\alpha \alpha}}+\frac{1}{k} \sum_{\beta}\left(\bar{J}^{E_{+}^{\beta \beta}}+\bar{J}^{E_{-}^{\beta \beta}}\right)\right)-\frac{1}{k} \sum_{\alpha>\beta} \bar{J}_{+}^{F_{+}^{\alpha \beta}} \bar{J}^{E_{-}^{\beta \alpha}} \\
& +\frac{1}{k} \sum_{\alpha<\beta} \bar{J}^{F_{+}^{\alpha \beta}} \bar{J}^{E_{+}^{\beta \alpha}}-\frac{1}{2 k} \sum_{\alpha}(-2 \alpha+1) \partial \bar{J}_{+}^{\alpha \alpha}
\end{aligned}
$$

Let us summarize this section. We started with a special choice of $\mathcal{N}=(2,2)$ superconformal algebra for the GL(N) $\times$ GL $(\mathrm{N})$ super WZNW model parameterized by deformation parameters $(\gamma, \bar{\gamma})$ in (3.19) and (3.28). We then have shown that the B-twisted topological field theory of this model is embedded in the free fermion resolution of the GL(N|N) WZNW model. Moreover the BRST-current as well as the ghost-number U(1)-current become affine Lie superalgebra currents. Also the ghost partner of the BRST-current has a nice expression in terms of Lie superalgebra currents. The full supergroup WZNW model is realized by coupling the bosons and fermions (2.41). Thus, the next step is to understand this boson-fermion interaction term. Since the world-sheet supercurrents are expressed in terms of the Lie superalgebra currents, it is a supersymmetric deformation and indeed it will turn out to be an F-term.

\subsection{The boson-fermion interaction term}

The boson-fermion interaction term $(2.42)$ is, as mentioned above, a supersymmetric deformation of our $\mathcal{N}=2 \mathrm{GL}(N) \times \mathrm{GL}(N)$ WZNW model. Since it is a potential term, we expect it to be an F-term and since we do not expect it to be exact, it should be a chiral F-term. On the $\operatorname{GL}(N \mid N)$ side the term takes the form

$$
S_{\text {pert }}=-\frac{1}{4 \pi k} \int_{\Sigma} d \tau d \sigma \operatorname{str}\left(\operatorname{Ad}\left(g_{B}\right)(\bar{b}) b\right)=\frac{1}{4 \pi k} \int_{\Sigma} d \tau d \sigma \bar{b}_{\beta \alpha} b_{\sigma \delta} \operatorname{tr}\left(E^{\alpha \beta} A^{-1} E^{\sigma \delta} B\right)
$$

where we have used the form of the $b$-matrices (3.5)

$$
b=b_{\alpha \beta} F_{+}^{\alpha \beta}, \quad \bar{b}=\bar{b}_{\beta \alpha} F_{-}^{\alpha \beta},
$$

and written

$$
g_{B}=\left(\begin{array}{cc}
A & 0 \\
0 & B
\end{array}\right) .
$$


The $E^{\alpha \beta}$ denotes the basis (3.15) of $\operatorname{gl}(\mathrm{N})$.

This boson-fermion interaction term is the F-term of a chiral field corresponding to chiral operator

$$
\phi=\operatorname{tr}\left(A^{-1} B\right)
$$

Indeed this field satisfies (2.28),

$$
G_{\gamma}^{+}(z) \operatorname{tr}\left(A^{-1} B\right)(w) \sim 0, \quad \bar{G}_{\bar{\gamma}}^{+}(z) \operatorname{tr}\left(A^{-1} B\right)(w) \sim 0
$$

using that in the bosonic subgroup $g(z, \bar{z})$ transforms as

$$
J_{a}(z) g(w) \sim \frac{-t_{a} g}{z-w}, \quad \bar{J}_{a}(z) g(w) \sim \frac{g t_{a}}{z-w},
$$

and the form of the generators in $\mathfrak{a}_{+}$(3.17). Further, it can be shown to be a Virasoro primary of dimension $(1 / 2,1 / 2)$.

Using (2.29) and the basis of $\mathfrak{a}_{-}$(3.17) we get the fermionic part of the chiral superfield

$$
\psi=-\frac{1}{k} \chi_{\delta \sigma} \operatorname{tr}\left(A^{-1} E^{\sigma \delta} B\right), \quad \bar{\psi}=\frac{1}{k} \bar{\chi}_{\alpha \beta} \operatorname{tr}\left(E^{\beta \alpha} A^{-1} B\right) .
$$

The F-term (2.30) takes the form

$$
F=-\frac{1}{k^{2}} \bar{\chi}_{\alpha \beta} \chi_{\delta \sigma} \operatorname{tr}\left(E^{\beta \alpha} A^{-1} E^{\sigma \delta} B\right)
$$

Using the identification of the ghosts (3.22) and (3.29) we see that the F-term is proportional to the boson-fermion interaction term (3.33) and using the chiral superfield $\Phi$ based on $\phi$ in (3.35) we have

$$
S_{\text {pert }}=\frac{k}{4 \pi} \int_{\Sigma} d \tau d \sigma \Phi
$$

Finally we would like to remark, that the construction gives us a wide range of chiral operators. One just needs to find a chiral field $\tilde{\phi}$ of conformal dimension $(1 / 2,1 / 2)$ satisfying

$$
G_{\gamma}^{+}(z) \tilde{\phi}(w, \bar{w}) \sim \bar{G}_{\bar{\gamma}}^{+}(\bar{z}) \tilde{\phi}(w, \bar{w}) \sim 0 .
$$

\subsection{The principal chiral field as a D-term}

Deformations that change the coefficient of the principal chiral field play an important role in the PSU $(1,1 \mid 2)$ supergroup sigma model where they describe Ramond-Ramond perturbations of the string theory $[22,23]$. In our $\operatorname{GL}(N \mid N)$ case the principal chiral field is given by

$$
S_{\text {kin }}=-\frac{k}{4 \pi} \int d^{2} z\left\langle g^{-1} \partial g, g^{-1} \bar{\partial} g\right\rangle \equiv \int d^{2} z \Phi_{\text {principal }} .
$$

Since this is a kinetic term, we expect it to be a D-term. Indeed, using the supergroup version of (3.37), (2.47) and $J=-k \partial g g^{-1}, \bar{J}=k g^{-1} \bar{\partial} g$ one can compute that

$$
\phi=-\frac{1}{4 \pi k}\left\langle\left(\begin{array}{c|c}
0 & \mathrm{Id} \\
\hline \mathrm{Id} & 0
\end{array}\right) g^{-1}\left(\begin{array}{c|c}
0 & \mathrm{Id} \\
\hline \mathrm{Id} & 0
\end{array}\right) J g \bar{J}\right\rangle,
$$


is the preimage of the principal chiral field:

$$
\Phi_{\text {principal }}=-\left\{G_{-1 / 2}^{+},\left[\bar{G}_{-1 / 2}^{+}, \phi\right]\right\}
$$

$\phi$ is not $G^{-}$closed and this is not an F-term, but rather a D-term. It is, however, $G^{+}+\bar{G}^{+}$ exact.

\section{Examples and applications}

In this section, we give some selected examples and applications.

\subsection{GL(2|2) and screening charges as chiral perturbations}

We start by considering the example of the GL(2|2) WZNW model from GL(2) $\times$ GL(2). In supergroup WZNW models one goes, in practice, beyond the free fermion realization and also introduces a Wakimoto free field realization for the bosonic subgroup. This is then supplemented with bosonic screening charges. In this section we will find that in addition to the boson-fermion interaction term also the bosonic screening charge is an F-term. As a consequence, the cohomology of $G^{-}$can be computed in free field theory. We start with some explicit formulae.

We denote the generators of $\operatorname{gl}(2) \oplus \operatorname{gl}(2)$ by $E_{ \pm}^{\alpha \beta}$ as before. Then the two $\operatorname{sl}(2) \mathrm{s}$ are generated by

$$
K_{ \pm}^{z}=E_{ \pm}^{11}-E_{ \pm}^{22} \quad, \quad K_{ \pm}^{+}=E_{ \pm}^{12} \quad \text { and } \quad K_{ \pm}^{-}=E_{ \pm}^{21}
$$

and the two central elements are

$$
K_{ \pm}^{0}=E_{ \pm}^{11}+E_{ \pm}^{22}
$$

The standard Manin decomposition. We are going to display the Manin decomposition and then the resulting supercurrents in the topological twisted theory as Lie superalgebra currents.

The bases for the Manin decomposition are

$$
\begin{aligned}
\mathfrak{a}_{+}=\{ & \frac{1}{2}\left(K_{+}^{0}+K_{+}^{z}+K_{-}^{0}+K_{-}^{z}\right), \frac{1}{2}\left(K_{+}^{0}-K_{+}^{z}+K_{-}^{0}-K_{-}^{z}\right), \\
\left.K_{+}^{+}+K_{-}^{+}, K_{+}^{-}+K_{-}^{-}\right\} & \mathfrak{a}_{-}=\left\{\frac{1}{4 k}\left(K_{+}^{0}+K_{+}^{z}-K_{-}^{0}-K_{-}^{z}\right)+\frac{1}{2 k^{2}}\left(K_{+}^{0}+K_{-}^{0}\right),\right. \\
& \left.\frac{K_{+}^{0}-K_{+}^{z}-K_{-}^{0}+K_{-}^{z}}{4 k}+\frac{1}{2 k^{2}}\left(K_{+}^{0}+K_{-}^{0}\right), \frac{1}{k} K_{+}^{-},-\frac{1}{k} K_{-}^{+}\right\} .
\end{aligned}
$$

The deformation parameter is

$$
\gamma=-\frac{1}{2 k}\left(K_{+}^{z}+K_{-}^{z}+(k+4) K_{+}^{0}+(4-k) K_{-}^{0}\right) .
$$


The supercurrents in the twisted theory in terms of superalgebra currents are

$$
\begin{aligned}
G_{\gamma}^{+}= & J^{F_{+}^{11}}+J^{F_{+}^{22}} \\
G_{\gamma}^{-}= & \frac{1}{4 k} J^{F_{-}^{11}}\left(J^{K_{+}^{z}}+J^{K_{+}^{0}}-J^{K_{-}^{z}}-J^{K_{-}^{0}}+\frac{2}{k}\left(J^{K_{+}^{0}}+J^{K_{-}^{0}}\right)\right) \\
& +\frac{1}{4 k} J^{F_{-}^{22}}\left(J^{K_{+}^{z}}-J^{K_{+}^{0}}-J^{K_{-}^{z}}+J^{K_{-}^{0}}+\frac{2}{k}\left(J^{K_{+}^{0}}+J^{K_{-}^{0}}\right)\right) \\
& \quad-\frac{1}{k} J^{F_{-}^{21}} J^{K_{-}^{+}}+\frac{1}{k} J^{F_{-}^{12}} J^{K_{+}^{-}}-\frac{3}{2 k} \partial J^{F_{-}^{11}}-\frac{1}{2 k} \partial J^{F_{-}^{22}} \\
U_{\gamma}= & \frac{1}{2} J^{K_{+}^{0}}-\frac{1}{2} J^{K_{-}^{0}}+\frac{1}{2 k} J^{K_{+}^{z}}+\frac{1}{2 k} J^{K_{-}^{z}} .
\end{aligned}
$$

Another Manin decomposition. Depending on the real form one wants to consider there are particularly good choices of $\mathcal{N}=2$ superconformal structure. We will now display a Manin triple that is well suited for $\mathrm{AdS}_{3} \times \mathrm{S}^{3} \times \mathrm{T}^{4}$. Again the relation to the supergroup WZNW model works nicely. The Manin decomposition is given by

$$
\begin{aligned}
& \mathfrak{a}_{+}=\left\{K_{+}^{0}+K_{-}^{0}, K_{+}^{z}-K_{-}^{z},-K_{+}^{+}+K_{-}^{-},-K_{+}^{-}+K_{-}^{+}\right\} \\
& \mathfrak{a}_{-}=\left\{\frac{2+k}{4 k^{2}} K_{+}^{0}-\frac{-2+k}{4 k^{2}} K_{-}^{0}, \frac{1}{4 k}\left(K_{+}^{z}+K_{-}^{z}\right),-\frac{1}{k} K_{-}^{+},-\frac{1}{k} K_{+}^{+}\right\}
\end{aligned}
$$

The deformation parameter $\gamma$ is in this case

$$
\gamma=-\frac{1}{2 k}\left(-K_{+}^{z}+K_{-}^{z}+(k+4) K_{+}^{0}+(-k+4) K_{-}^{0}\right) .
$$

This changes the central charge from 12 to zero.

Further, the fermions in the twisted theory are identified with the $b c$-ghosts of the free fermion realization of the supergroup as follows,

$$
\begin{aligned}
& b_{11}=\chi_{4}, \quad b_{12}=\frac{1}{2}\left(\chi_{1}+\chi_{2}\right), \quad b_{21}=\frac{1}{2}\left(-\chi_{1}+\chi_{2}\right), \quad b_{22}=-\chi_{3}, \\
& c^{11}=\chi^{4}, \quad c^{12}=\chi^{1}+\chi^{2}, \quad c^{21}=-\chi^{1}+\chi^{2}, \quad c^{22}=-\chi^{3} \text {. }
\end{aligned}
$$

Here $\chi_{i}\left(\chi^{i}\right)$ is the fermion corresponding to the $i$-th generator of $\mathfrak{a}_{+}\left(\mathfrak{a}_{-}\right)$, see $(2.10)$. The supercurrents are identified as

$$
\begin{aligned}
G_{\gamma}^{+}=- & J^{F_{+}^{12}}+J^{F_{+}^{21}} \\
G_{\gamma}^{-}=- & \frac{1}{4 k} J^{F_{-}^{21}}\left(J^{K_{+}^{z}}+J^{K_{+}^{0}}+J^{K_{-}^{z}}-J^{K_{-}^{0}}+\frac{2}{k}\left(J^{K_{+}^{0}}+J^{K_{-}^{0}}\right)\right) \\
& +\frac{1}{4 k} J^{F_{-}^{12}}\left(-J^{K_{+}^{z}}+J^{K_{+}^{0}}-J^{K_{-}^{z}}-J^{K_{-}^{0}}+\frac{2}{k}\left(J^{K_{+}^{0}}+J^{K_{-}^{0}}\right)\right) \\
& \quad-\frac{1}{k} J^{F_{-}^{22}} J^{K_{-}^{+}}+\frac{1}{k} J^{F_{-}^{11}} J^{K_{+}^{+}}-\frac{3}{2 k} \partial J^{F_{-}^{12}}+\frac{1}{2 k} \partial J^{F_{-}^{21}} \\
U_{\gamma}= & \frac{1}{2} J^{K_{+}^{0}}-\frac{1}{2} J^{K_{-}^{0}}-\frac{1}{2 k} J^{K_{+}^{z}}+\frac{1}{2 k} J^{K_{-}^{z}} .
\end{aligned}
$$


The anti-holomorphic side is analogous if we choose the Manin triple for the antiholomorphic side to be different, but only by an automorphism. It is

$$
\begin{aligned}
& \mathfrak{a}_{+}=\left\{-K_{+}^{0}-K_{-}^{0},-K_{+}^{z}+K_{-}^{z}, K_{+}^{-}-K_{-}^{+}, K_{+}^{+}-K_{-}^{-}\right\} \\
& \mathfrak{a}_{-}=\left\{-\frac{2+k}{4 k^{2}} K_{+}^{0}+\frac{-2+k}{4 k^{2}} K_{-}^{0},-\frac{1}{4 k}\left(K_{+}^{z}+K_{-}^{z}\right), \frac{1}{k} K_{-}^{-}, \frac{1}{k} K_{+}^{-}\right\}
\end{aligned}
$$

The deformation parameter coincides with the one of the chiral half, $\bar{\gamma}=\gamma$.

Screening charges as chiral perturbations. We will now show that the screening charges - both the boson-fermion interaction terms and the bosonic screening charges can be seen as chiral perturbations.

For the standard Manin decomposition we have already seen that the boson-fermion interaction terms can be seen as a chiral perturbation (3.40). This also happens in the case of the alternative Manin decomposition, but the chiral field generating the multiplet has to be changed from (3.35) by conjugation with a constant matrix

$$
\phi=\frac{k}{4 \pi} \operatorname{tr}\left(\left(\begin{array}{cc}
0 & -1 \\
1 & 0
\end{array}\right) A^{-1}\left(\begin{array}{cc}
0 & 1 \\
-1 & 0
\end{array}\right) B\right) .
$$

Then the corresponding F-term $\Phi=-\left\{G_{-1 / 2}^{-},\left[\bar{G}_{-1 / 2}^{-}, \phi\right]\right\}$ gives the boson-fermion interaction terms.

We can however go one step further. Looking at the free fermion resolution (2.42) we also have interaction terms in the WZNW action for the bosonic subgroup. We can now go to the first order formalism for the bosonic subgroup and write the bosonic Lagrangian as first order kinetic terms plus bosonic screening charges. Our claim is that the screening charges are also chiral perturbations. We will now show this in the case of the new Manin decomposition.

The action for the bosonic subgroup (2.42) renormalized with the metric (3.9) takes the form:

$$
\begin{aligned}
& S_{\text {ren }}^{\mathrm{WZNW}}\left[g_{B}\right]=-\frac{1}{4 \pi} \int_{\Sigma} d^{2} z\left\langle g^{-1} \partial g, g^{-1} \bar{\partial} g\right\rangle_{\mathrm{ren}}-\frac{1}{24 \pi} \int_{B}\left\langle g^{-1} d g,\left[g^{-1} d g, g^{-1} d g\right]\right\rangle_{\mathrm{ren}} \\
&=\frac{1}{2 \pi} \int_{\Sigma} d^{2} z[-(k-2) \partial \phi_{+}^{0} \bar{\partial} \phi_{+}^{0}+(k+2) \partial \phi_{-}^{0} \bar{\partial} \phi_{-}^{0}-2 \partial \phi_{+}^{0} \bar{\partial} \phi_{-}^{0}-2 \partial \phi_{-}^{0} \bar{\partial} \phi_{+}^{0} \\
&-(k-2) \partial \phi_{+} \bar{\partial} \phi_{+}-(k-2) \partial \bar{\gamma}_{+} \bar{\partial} \gamma_{+} e^{-2 \phi_{+}} \\
&\left.+(k+2) \partial \phi_{-} \bar{\partial} \phi_{-}+(k+2) \partial \bar{\gamma}_{-} \bar{\partial} \gamma_{-} e^{-2 \phi_{-}}\right]
\end{aligned}
$$

where we have chosen a parametrization of the Lie group valued field $g_{B}$ via a Gauss decomposition

$$
g_{B}=e^{\gamma_{+} K_{+}^{+}+\gamma_{-} K_{-}^{+}} e^{\phi_{+}^{0} K_{+}^{0}+\phi_{-}^{0} K_{-}^{0}+\phi_{+} K_{+}^{z}+\phi_{-} K_{-}^{z}} e^{\bar{\gamma}_{+} K_{+}^{-}+\bar{\gamma}_{-} K_{-}^{-}} .
$$

We now introduce the auxiliary field $\beta_{ \pm}, \bar{\beta}_{ \pm}$. Remembering corrections from the quantum measure - which essentially cancels the shift in levels for the two SU(2) factors we get for 
the upper SL(2)-part of the action

$$
S_{+}=\frac{1}{2 \pi} \int_{\Sigma} d^{2} z\left(-k \partial \phi_{+} \bar{\partial} \phi_{+}+\beta_{+} \bar{\partial} \gamma_{+}+\bar{\beta} \partial \bar{\gamma}_{+}+\frac{1}{k-2} \beta \bar{\beta} e^{2 \phi_{+}}+\frac{1}{4} \sqrt{h} \mathcal{R}^{(2)} \phi_{+}\right),
$$

and similarly for the lower SL(2) part. The bosonic currents in this first order formalism are

$$
\begin{aligned}
& J_{B}^{K_{+}^{z}}=-2 k \partial \phi_{+}+2: \gamma_{+} \beta_{+}: \\
& J_{B}^{K_{+}^{+}}=\beta_{+}, \\
& J_{B}^{K_{+}^{-}}=2 k \partial \phi_{+} \gamma_{+}-(k-2) \partial \gamma_{+}-: \beta_{+} \gamma_{+} \gamma_{+}:,
\end{aligned}
$$

they can be inserted in the free fermion resolution (3.11) to give an expression for $G^{-}$ in (4.12). For completeness the anti-holomorphic currents are:

$$
\begin{aligned}
& \bar{J}_{B}^{K_{+}^{z}}=2 k \bar{\partial} \phi_{+}-2: \bar{\gamma}_{+} \bar{\beta}_{+}:, \\
& \bar{J}_{B}^{K_{+}^{+}}=-2 k \bar{\partial} \phi_{+} \bar{\gamma}_{+}+(k-2) \bar{\partial} \bar{\gamma}_{+}+: \bar{\beta}_{+} \bar{\gamma}_{+} \bar{\gamma}_{+}:, \\
& \bar{J}_{B}^{K_{+}^{-}}=-\bar{\beta}_{+} .
\end{aligned}
$$

Using this, we compute that the screening charge $\Phi_{B}=\beta \bar{\beta} e^{2 \phi_{+}}$is the F-term of the supermultiplet obtained from the chiral field

$$
\phi_{B}=\frac{k^{2}}{k-2} c^{11} \bar{c}^{11} e^{2 \phi_{+}}
$$

and similarly for the screening charge of the lower SL(2) part.

In conclusion, we have shown that the whole action for the supergroup can be written as simple first order kinetic terms (and background charges) plus interaction terms in form of screening charges that can be written as chiral F-terms. These are by construction exact in $G^{-}$(and $\bar{G}^{-}$). A practical consequence is that the cohomology of $G^{-}$(and $\bar{G}^{-}$) can be computed in free field theory.

\subsection{Comparison to string theory on $\operatorname{AdS}_{3} \times \mathrm{S}^{3} \times \mathrm{T}^{4}$}

In this section we show how our story is related to type IIB string theory on $\operatorname{AdS}_{3} \times \mathrm{S}^{3} \times \mathrm{T}^{4}$.

String theory on $\mathrm{AdS}_{3} \times \mathrm{S}^{3} \times \mathrm{T}^{4}$ consists of bosons parameterizing the space related to the group $\mathrm{SL}(2) \times \mathrm{SU}(2) \times \mathrm{U}(1)^{4}$, their corresponding fermionic partners and the string ghosts. The total central charge is zero.

Let us compare this to our approach for the case of the group $\mathrm{U}(1,1 \mid 2)_{-k} \times \mathrm{U}(1 \mid 1) \times$ $\mathrm{U}(1 \mid 1)$. The bosonic group that forms our starting point is then $\mathrm{U}(1,1) \times \mathrm{U}(2) \times \mathrm{U}(1)^{4}$, and further we have the corresponding decoupled fermionic partners. After the deformation with $\gamma$, but before twisting the theory, this system also has central charge zero. Remember that $\mathrm{U}(1,1)$ is equivalent to $\mathrm{U}(1) \times \mathrm{SL}(2)$. Thus the bosonic group that we start with has two U(1)s plus their fermionic partners more than on the string side. Bosonizing the fermions we thus have three extra scalars with various background charges due to the deformation. On the string theory side we, however, have the superconformal ghosts 
which consist of a $\beta \gamma$ - and a $b c$-ghost system. Bosonizing also gives three scalars with background charges. So the field content is basically the same in string theory and in our case of $\mathrm{U}(1,1 \mid 2)_{-k} \times \mathrm{U}(1 \mid 1) \times \mathrm{U}(1 \mid 1)$. We will now show how to precisely embed string theory into our story.

$\mathbf{A d S}_{\mathbf{3}} \times \mathbf{S}^{\mathbf{3}}$. Let us first consider the string theory on $\mathrm{AdS}_{3} \times \mathrm{S}^{3} \times \mathrm{T}^{4}$ in detail following [24]. The string theory is described by the $\mathcal{N}=1$ WZNW model on $\mathrm{SL}(2)_{-k} \times \mathrm{SU}(2)_{k} \times \mathrm{U}(1)^{4}$ plus superconformal ghosts. The negative level in $\mathrm{SL}(2)$ gives the correct sign on the Cartan kinetic term and makes sure the total central charge is zero. After decoupling the fermions the $\mathcal{N}=1$ supercurrent schematically takes the form (suppressing factors of $-k$ )

$$
G_{\text {string }}^{\mathcal{N}=1}=J_{a} \chi^{a}-\frac{1}{6} f^{a b}{ }_{c} \chi_{a} \chi_{b} \chi^{c}
$$

where the indices $a, b, c$ run over the entire algebra, $J_{a}$ are the currents, $\chi^{a}$ are the fermion partners from (2.1), and $f^{a b}{ }_{c}$ are the structure constants of the entire algebra. Indices are raised and lowered using the metric. Note that this form is only unique up to field redefinitions of the fermions that preserve the OPEs. Indeed, given such an isometry of the fermions the action is left invariant, but $G^{\mathcal{N}=1}$ changes its form.

The string theory stress energy tensor splits in three parts corresponding to the $\mathrm{AdS}_{3} \times$ $\mathrm{S}^{3}, \mathrm{~T}^{4}$ and the ghosts

$$
T_{\text {string }}=T_{\text {string }}^{\mathrm{AdS}_{3} \times \mathrm{S}^{3}}+T_{\text {string }}^{\mathrm{T}^{4}}+T_{\text {string }}^{\text {ghosts }},
$$

and similarly for the rest of the superalgebra.

In appendix B of $[24]$ the $\mathrm{U}(1)$ current, $U_{\text {string }}^{\mathrm{AdS}_{3} \times \mathrm{S}^{3}}+U_{\text {string }}^{\mathrm{T}^{4}}$, leading to the chiral ring of string theory is written. Given $U_{\text {string }}$ we can find $G_{\text {string }}^{ \pm}$using that they have opposite $\mathrm{U}(1)$ charge and $G_{\text {string }}^{\mathcal{N}=1}=G_{\text {string }}^{+}+G_{\text {string. }}^{-}$. Here and in the following we write the basis of $\mathrm{SU}(2)$ in the form of $\mathrm{SL}(2)$, i.e. we use generators

$$
t^{ \pm}=t^{x} \pm i t^{y}
$$

We will use our notation for the basis of $\mathrm{SL}(2) \times \mathrm{SU}(2)$ as in (4.1) i.e. $K_{+}^{ \pm, z}$ spans $\mathrm{SL}(2)$ and $K_{-}^{ \pm, z} \operatorname{span} \mathrm{SU}(2)$.

The $\mathrm{AdS}_{3} \times \mathrm{S}^{3}$ part of the $\mathrm{U}(1)$ from [24] then reads

$$
U_{\text {string }}^{\mathrm{AdS}_{3} \times S^{3}}=\frac{1}{k} J^{K_{+}^{z}}-\frac{1}{k} J^{K_{-}^{z}}+\frac{1}{2 k} \chi^{K_{+}^{z}} \chi^{K_{-}^{z}}-\frac{k+2}{k^{2}} \chi^{K_{+}^{+}} \chi^{K_{+}^{-}}+\frac{k-2}{k^{2}} \chi^{K_{-}^{+}} \chi^{K_{-}^{-}} .
$$

We can now ask what the expressions for $G_{\text {string, } \operatorname{AdS}_{3} \times \mathrm{S}^{3}}^{ \pm}$are. To find these we simply note that the $\mathrm{U}(1)$ current corresponds to the Manin decomposition

$$
\begin{aligned}
& \mathfrak{a}_{+}=\left\{K_{+}^{z}-K_{-}^{z}, K_{-}^{-},-K_{+}^{-}\right\} \\
& \mathfrak{a}_{-}=\left\{-\frac{1}{4 k}\left(K_{+}^{z}+K_{-}^{z}\right), \frac{1}{k} K_{-}^{+}, \frac{1}{k} K_{+}^{+}\right\} .
\end{aligned}
$$

The currents $G_{\text {string, }}^{ \pm} \mathrm{AdS}_{3} \times \mathrm{S}^{3}$ then follow directly from (2.12). Note that this Manin decomposition is very symmetric, and the $G^{+}$- and $G^{-}$-chiral ring are dual (conjugately related). 
Further, $\mathfrak{a}_{-}$is exactly the same as we have in the $\mathrm{U}(1,1 \mid 2)_{-k}$ case with the overall $U(1) \mathrm{s}$ removed for the alternative Manin decomposition in (4.8). So, if we identify our fermions with the corresponding string fermions

$$
\chi_{i}^{\text {string }}=\chi_{i}, \quad \chi_{\text {string }}^{i}=\chi^{i}
$$

we get the same $G^{-}$current, and actually also the same $U$ current

$$
\begin{aligned}
& G_{\text {string, } \mathrm{AdS}_{3} \times \mathrm{S}^{3}}^{-}=\left.G^{-}\right|_{\mathrm{SL}(2) \times \mathrm{SU}(2)}, \\
& U_{\text {string, } \mathrm{AdS}_{3} \times \mathrm{S}^{3}}=\left.U\right|_{\mathrm{SL}(2) \times \mathrm{SU}(2)} .
\end{aligned}
$$

The currents have here been identified by going back to the original basis $K_{ \pm}^{ \pm, z}$

$$
J_{\text {string }}^{K_{ \pm}^{ \pm, z}}=J^{K_{ \pm}^{ \pm, z}}
$$

In this original basis the identification of the fermions are not trivial since the Manin bases $\mathfrak{a}_{+}$are different ${ }^{4}$ i.e. $x_{\text {string }}^{i}=x^{i}$, but $x_{i}^{\text {string }} \neq x_{i}$. The non-trivial identifications of the fermions are

$$
\chi_{\text {string }}^{K_{-}^{-}}=\chi^{K_{-}^{-}}-\chi^{K_{+}^{+},} \quad \chi_{\text {string }}^{K_{+}^{-}}=\chi^{K_{+}^{-}}-\chi^{K_{-}^{+}} .
$$

It is clear from (4.28) that this identification preserves the standard OPEs and hence is an isometry leaving the action invariant. This means that $G_{\text {string }}^{\mathcal{N}=1}$ does not get mapped into $G^{\mathcal{N}=1}=G^{+}+G^{-}$, but they are identical up to the isometry of the fermions. This also means that the $G_{\text {string }}^{+}$does not get mapped into $G^{+}$and the difference is

$$
G_{\text {string, } \mathrm{AdS}_{3} \times \mathrm{S}^{3}}^{+}=\left.G^{+}\right|_{\mathrm{SL}(2) \times \mathrm{SU}(2)}+\frac{1}{k} J^{K_{+}^{+}} \chi^{K_{-}^{+}}-\frac{1}{k} J^{K_{-}^{+}} \chi^{K_{+}^{+}}-\frac{1}{k^{2}}\left(\chi^{K_{+}^{z}}-\chi^{K_{-}^{z}}\right) \chi^{K_{+}^{+}} \chi^{K_{-}^{+}} .
$$

In other words, we have constructed another $\mathcal{N}=2$ supersymmetry of the string theory that only differ in the $G^{+}$current. This difference is important to achieve that $G^{+}$after deformation and twist is a current of the $\mathrm{U}(1,1 \mid 2)_{-k}$ model.

Further, we have to do the $\gamma$ deformation (4.9). For the $\mathrm{AdS}_{3}$ part this is just like the type of spectral flows suggested in [25]. This is then extended to also include the $S^{3}$ sector and to deform the fermions to preserve the $\mathcal{N}=2$ supersymmetry. This part of the deformation does not change the central charge. Let us again stress that the deformation only changes the weights in the chiral ring.

Ghosts and $\mathbf{T}^{4}$. We want to compare the ghosts and $\mathrm{T}^{4}$ of string theory with the U(1)s in the $\mathrm{U}(1,1 \mid 2)_{-k} \times \mathrm{U}(1 \mid 1) \times \mathrm{U}(1 \mid 1)$. To study this we need to add the generators of the bosonic bases of $\mathrm{U}(1 \mid 1)$ to the Manin decomposition (4.8). This means that the $\mathcal{N}=2$

\footnotetext{
${ }^{4}$ Since $\mathfrak{a}_{+}$is simpler in string theory than in our case, the supersymmetry algebra have a two-dimensional space of deformations instead of the one-dimensional in our case.
} 
supersymmetric theory of the $\mathrm{U}(1) \mathrm{s}$ is described by $J_{i}, J^{i}$ and the superpartners $\chi_{i}, \chi^{i}$ where $i=1,5,6$. The supersymmetric algebra after the deformation given by (4.9) is then

$$
\begin{aligned}
\left.G_{\gamma}^{+}\right|_{\mathrm{U}(1) \mathrm{s}} & =J_{i} \chi^{i}+\partial\left(2 \chi^{1}-\chi^{5}-\chi^{6}\right), \\
\left.G_{\gamma}^{-}\right|_{\mathrm{U}(1) \mathrm{s}} & =J^{i} \chi_{i}+\partial\left(\chi_{1}-\frac{1}{2} \chi_{5}-\frac{1}{2} \chi_{6}\right), \\
\left.U_{\gamma}\right|_{\mathrm{U}(1) \mathrm{s}} & =\chi^{i} \chi_{i}+J_{1}-2 J^{1}-\frac{1}{2} J_{5}+J^{5}-\frac{1}{2} J_{6}+J^{6}, \\
\left.T_{\gamma}\right|_{\mathrm{U}(1) \mathrm{s}} & =J_{i} J^{i}+\frac{1}{2}\left(\partial \chi^{i} \chi_{i}-\chi^{i} \partial \chi_{i}\right)+\partial\left(\frac{1}{2} J_{1}+J^{1}-\frac{1}{4} J_{5}-\frac{1}{2} J^{5}-\frac{1}{4} J_{6}-\frac{1}{2} J^{6}\right) .
\end{aligned}
$$

Here we have performed a rescaling of the fermions and currents to absorb the levels, but keeping the kinetic terms. The levels can in principle be different for the U(1|1)s.

We now turn to the $\mathrm{T}^{4}$ part of string theory. To anticipate the embedding into our theory we denote the currents by $\hat{J}_{i}, \hat{J}^{i}$ and the fermions by $\hat{\chi}_{i}, \hat{\chi}^{i}$ where $i=5,6$. The $\mathcal{N}=2$ algebra is given standardly by. ${ }^{5}$

$$
\begin{aligned}
& G_{\text {string, } \mathrm{T}^{4}}^{+}=\hat{J}_{i} \hat{\chi}^{i}, \\
& G_{\text {string, } \mathrm{T}^{4}}=\hat{J}^{i} \hat{\chi}_{i}, \\
& U_{\text {string, } \mathrm{T}^{4}}=\hat{\chi}^{i} \hat{\chi}_{i}, \\
& T_{\text {string, } \mathrm{T}^{4}}=\hat{J}_{i} \hat{J}^{i}+\frac{1}{2}\left(\partial \hat{\chi}^{i} \hat{\chi}_{i}-\hat{\chi}^{i} \partial \hat{\chi}_{i}\right) .
\end{aligned}
$$

Finally, we consider the ghost system which consists of a $\beta \gamma$-system of central charge 11 and a $b c$-system of central charge -26 . The $\mathcal{N}=1$ supercurrent of the ghost system is given in e.g. [26]. ${ }^{6}$ Without bosonization the only possible extension to an $\mathcal{N}=2$ algebra (without bosonization and up to swapping of $G^{ \pm}$) takes the following form

$$
\begin{aligned}
& G_{\text {string, ghost }}^{+}=-2 i b \gamma \\
& G_{\text {string, ghost }}^{-}=-i(\partial \beta) c-i \frac{3}{2} \beta \partial c, \\
& U_{\text {string, ghost }}=-2: b c:-3: \gamma \beta:, \\
& T_{\text {string, ghost }}=-: \partial b c:-2: b \partial c:-\frac{1}{2}: \beta \gamma:-\frac{3}{2}: \beta \partial \gamma: .
\end{aligned}
$$

We now bosonize the ghosts into three scalars, the $b c$-system in the ordinary way $b=e^{-\rho_{1}}$, $c=e^{\rho_{1}}$ and the $\beta \gamma$-system we bosonize oppositely as normal $\gamma=i e^{-\rho_{2}+\rho_{3}} \partial \rho_{3}, \beta=i e^{\rho_{2}-\rho_{3}}$. The bosonized currents are

$$
\begin{aligned}
G_{\text {string, ghost }}^{+} & =2 e^{-\rho_{1}-\rho_{2}+\rho_{3}} \partial \rho_{3}, \\
G_{\text {string, ghost }}^{-} & =e^{\rho_{1}+\rho_{2}-\rho_{3}} \partial\left(\frac{3}{2} \rho_{1}+\rho_{2}-\rho_{3}\right) \\
U_{\text {string, ghost }} & =\partial\left(2 \rho_{1}+3 \rho_{2}\right), \\
T_{\text {string, ghost }} & =\frac{1}{2} \partial \rho_{1} \partial \rho_{1}+\frac{3}{2} \partial^{2} \rho_{1}-\frac{1}{2} \partial \rho_{2} \partial \rho_{2}+\frac{1}{2} \partial \rho_{3} \partial \rho_{3}+\partial^{2} \rho_{2}+\frac{1}{2} \partial^{2} \rho_{3} .
\end{aligned}
$$

\footnotetext{
${ }^{5}$ Note that [24] has an extra overall $i$ in the $U$ current.

${ }^{6}$ We correct the formula with an $i$.
} 
To be able to compare we re-fermionize this system. We define $\hat{\chi}_{1}=\frac{3}{2} e^{\rho_{1}+\rho_{2}-\rho_{3}}, \hat{\chi}^{1}=$ $\frac{2}{3} e^{-\rho_{1}-\rho_{2}+\rho_{3}}, \hat{J}_{1}=3\left(\rho_{1}+\rho_{2}\right)$ and $\hat{J}^{1}=\frac{1}{3}\left(-\rho_{2}+\rho_{3}\right)$. We then arrive at

$$
\begin{aligned}
& G_{\text {string, ghost }}^{+}=\hat{J}_{1} \hat{\chi}^{1}+3 \partial \hat{\chi}^{1}, \\
& G_{\text {string, ghost }}^{-}=\hat{J}^{1} \hat{\chi}_{1}+\partial \hat{\chi}_{1}, \\
& U_{\text {string, ghost }}=\hat{\chi}^{1} \hat{\chi}_{1}+\partial \hat{J}_{1}-3 \partial \hat{J}^{1}, \\
& T_{\text {string, ghost }}=\hat{J}_{1} \hat{J}^{1}+\frac{1}{2}\left(\partial \hat{\chi}^{1} \hat{\chi}_{1}-\hat{\chi}^{1} \partial \hat{\chi}_{1}\right)+\frac{1}{2} \partial \hat{J}_{1}+\frac{3}{2} \partial \hat{J}^{1} .
\end{aligned}
$$

Note that this could also easily be put into a form that is symmetric in $G^{ \pm}$again showing that the $G^{ \pm}$-chiral rings of string theory are in one-to-one correspondence.

We can now get a direct match of our superalgebra (4.33) for the U(1)s and the string superalgebra for the ghosts and $\mathrm{T}^{4}$ in equations (4.34) and (4.37) using the dictionary

$$
\begin{array}{ll}
\hat{\chi}_{(i)}=A \chi_{(i)}, & \hat{\chi}^{(i)}=\left(A^{-1}\right)^{T} \chi^{(i)}, \\
\hat{J}_{(i)}=A J_{(i)}, & \hat{J}^{(i)}=\left(A^{-1}\right)^{T} J^{(i)},
\end{array}
$$

where $\chi_{(i)}^{T}$ is the vector $\left(\chi_{1}, \chi_{5}, \chi_{6}\right)$ etc. and $A$ is the matrix

$$
A=\left(\begin{array}{ccc}
1 & -\frac{1}{2} & -\frac{1}{2} \\
\frac{1}{2} & 1 & 0 \\
\frac{1}{2} & 0 & 1
\end{array}\right) .
$$

To sum up, we have shown in this section that the superalgebra we have after deformation, i.e. right before twisting, in the case of $\mathrm{U}(1,1 \mid 2)_{-k} \times \mathrm{U}(1 \mid 1) \times \mathrm{U}(1 \mid 1)$ only differs from the standard string theory algebra in the $G^{+}$part, and that our algebra is simply another choice of $\mathcal{N}=2$ algebra.

\subsection{Boundary actions and the Warner problem}

In this section, we want to understand boundary actions in supergroup WZNW models using methods of world-sheet supersymmetric theories. Warner explained how to find Btype boundary actions in superconformal field theories [7], we use [27, 28] as references. Boundary conformal field theory on supergroups has been investigated in [5, 18, 23, 29-32].

Lie supergroup bulk WZNW models can be well treated in the free fermion formalism. A similar method is desired for the boundary theories. The problem is to find the appropriate boundary action. So far only in GL(1|1) [5] and in $\operatorname{OSP}(1 \mid 2)$ [6] this problem could be solved. On the other hand, knowing the boundary action was essential in solving these models.

Boundary conformal field theory in WZNW models is characterized by boundary conditions that preserve the current algebra in addition to conformal symmetry. This is the case if and only if the holomorphic and anti-holomorphic currents are glued together at the boundary with a metric preserving automorphism $\omega$ of the underlying horizontal subalgebra

$$
J(z)=\omega(\bar{J}(\bar{z})) \quad \text { for } z=\bar{z} .
$$


A consequence of these conditions is that the group valued field that describes the sigma model maps the boundary of the world-sheet to a twisted (super) conjugacy class $C_{a}^{\omega}$,

$$
C_{a}^{\omega}=\left\{g=h a \omega\left(h^{-1}\right) \mid h \in G\right\}
$$

The constant element $a$ is parameterizing the position of the brane.

Now, we saw that the bulk GL(N|N) WZNW model can be obtained by twisting an $\mathcal{N}=(2,2)$ superconformal model. Moreover, the boson-fermion interaction term is an Fterm. In this section, we want to consider the boundary theories with gluing automorphism $\omega$ being minus one times the transpose in our matrix representation. This implies that our matrix valued fields $A$ and $B$ in GL(N) have the form

$$
A=C A_{0} C^{t} \quad \text { and } \quad B=D B_{0} D^{t}
$$

for some GL(N) valued fields $C$ and $D$ and constants matrices $A_{0}$ and $B_{0}$.

We want to find a boundary action that preserves the superconformal symmetry. This problem is often referred to as the Warner problem. Its solution requires additional fermionic boundary degrees of freedom as well as a factorization of the super potential into boundary super potentials. Let us review this situation for B-branes in Landau-Ginzburg models. The world-sheet of a Landau-Ginzburg model has two complex fermionic coordinates. The action of the model is given by a D-term and an F-term, $\mathcal{L}=\mathcal{L}_{D}+\mathcal{L}_{F}$, which are both by construction invariant under supersymmetry transformations. If one integrates the fermionic coordinates, the F-term looks as follows

$$
\mathcal{L}_{F}=\frac{1}{4}\left|W^{\prime}\right|^{2}+\frac{1}{2} W^{\prime \prime} \psi_{+} \psi_{-}-\frac{1}{2} \bar{W}^{\prime \prime} \bar{\psi}_{+} \bar{\psi}_{-} .
$$

A simple choice of D-term is, after integrating the fermionic coordinates, the Lagrangian of free bosons and fermions

$$
\mathcal{L}_{D}=\partial \bar{\phi} \bar{\partial} \phi+\partial \phi \bar{\partial} \bar{\phi}+\bar{\psi}_{-} \partial \psi_{-}+\psi_{-} \partial \bar{\psi}_{-}+\bar{\psi}_{+} \bar{\partial} \psi_{+}+\psi_{+} \bar{\partial} \bar{\psi}_{+}
$$

There exist two families of boundary conditions that preserve half of the supersymmetry, which are called A- and B-boundary conditions. We are interested in the second case. First, if one sets the super potential $W$ to zero, B-type supersymmetry is preserved by introducing the following boundary term

$$
S_{0, \text { bdy }}=\frac{i}{4} \int d \tau \bar{\theta} \eta-\bar{\eta} \theta
$$

where $\eta=\psi_{-}+\psi_{+}$and $\theta=\psi_{-}-\psi_{+}$. In this free theory this amounts to the boundary conditions $\psi_{-}=\psi_{+}$and $\bar{\psi}_{-}=\bar{\psi}_{+}$for the fermions. For non-zero super potential $W$, the supersymmetry variation of the action gives a boundary term of the form

$$
\delta_{\text {susy }}\left(S+S_{0, \text { bdy }}\right)=\frac{i}{2} \int d \tau \epsilon \bar{\eta} \bar{W}+\bar{\epsilon} \eta W
$$


Finding a boundary term whose variation cancels this contribution is the Warner problem. Its solution is given by introducing a fermionic boundary super field. After integrating the odd world-sheet coordinates the boundary term is

$$
S_{\mathrm{bdy}}=\int d \tau i \bar{\pi} \partial_{\tau} \pi-\frac{1}{2} \bar{J} J-\frac{1}{2} \bar{E} E+\frac{i}{2} \pi \eta J^{\prime}+\frac{i}{2} \bar{\pi} \bar{\eta} \bar{J}^{\prime}-\frac{1}{2} \bar{\pi} \eta E^{\prime}-\frac{1}{2} \pi \bar{\eta} \bar{E}^{\prime} .
$$

Here $J(\phi)$ and $E(\phi)$ are boundary potentials, prime denotes derivative with respect to $\phi$, and $\pi$ is the new boundary fermion. Its variation under supersymmetry is

$$
\delta_{\text {susy }} \pi=-i \epsilon \bar{J}-\bar{\epsilon} E, \quad \delta_{\text {susy }} \bar{\pi}=i \bar{\epsilon} J-\epsilon \bar{E} .
$$

The total action is now invariant under supersymmetry variation if and only if

$$
W=E J+\text { constant } .
$$

In the case of the $\mathrm{U}(1) \times \mathrm{SU}(2)$ WZNW model a superfield formulation of the theory is known [33]. This motivates us to use an analogous method to solve the Warner problem in our case. We already saw in the last section that the bulk superpotential is

$$
W(A, B)=\operatorname{tr}\left(A^{-1} B\right), \quad \bar{W}=0 .
$$

Here $A$ and $B$ are the two GL(N) (matrix) valued fields describing the GL $(\mathrm{N}) \times \mathrm{GL}(\mathrm{N})$ WZNW model. Recall that we choose gluing conditions for the currents that force the matrix valued fields to have the form $A=C A_{0} C^{t}$ and $B=D B_{0} D^{t}$ at the boundary, where $C$ and $D$ are some matrix valued fields.

Further, since $\bar{W}=0$, we should have $\bar{J}=\bar{E}=0$. The field $\eta$ is in our case $\eta=b=\bar{b}^{t}$ and is a $\operatorname{gl}(\mathrm{N})$ matrix valued fermionic field. Since we identify $b$ with $\bar{b}^{t}$, we also want to identify $\bar{\pi}$ with $\pi$ in some way. Since $\bar{\pi} \pi$ should be a scalar field we take

$$
i \bar{\pi}=\operatorname{tr}\left(B_{0} \pi^{t} A_{0}^{-1} \cdot\right)
$$

for two constant $\mathrm{GL}(\mathrm{N})$ matrices $A_{0}$ and $B_{0}$. Then the supersymmetry variation (4.46) forces to also identify

$$
J=\operatorname{tr}\left(B_{0} E^{t} A_{0}^{-1} \cdot\right) .
$$

We define $E=C^{-1} D$ for two GL(N) valued boundary fields $C$ and $D$, such that the invariant vector fields act as

$$
\partial_{\sigma \delta} E=C^{-1} E^{\sigma \delta} D .
$$

Moreover, we have $J E=W$ from (4.40) as required. Then the boundary action (4.45) takes the form

$$
S_{\mathrm{bdy}}=\int d \tau \operatorname{tr}\left(B_{0} \pi^{t} A_{0}^{-1} \partial_{\tau} \pi\right)-\frac{i}{2} \operatorname{tr}\left(B_{0} D^{t} b^{t}\left(C^{-1}\right)^{t} A_{0}^{-1} \pi\right)+\frac{i}{2} \operatorname{tr}\left(B_{0} \pi^{t} A_{0}^{-1} C^{-1} b D\right) .
$$

We believe that this is the correct action of the boundary model, and it is indeed in the example of GL(1|1) [5]. In order to prove this statement rigourously one has to show that the boundary potential is a screening charge for the currents. We have not proven this in general, but it is straightforward to address this issue in the example one wants to study. 


\section{Summary and outlook}

We have shown that the B-twist of the world-sheet supersymmetric $\operatorname{GL}(N) \times \operatorname{GL}(N)$ WZNW model perturbed by a truly marginal operator, an F-term, is embedded in the $\mathrm{GL}(N \mid N)$ WZNW model. Moreover, the supercurrents, as well as the U(1)-current, are expressions in terms of Lie superalgebra currents. Further, we have seen that the principal chiral field is a D-term.

We then applied these results. In the example of the GL(2|2) WZNW model we have shown that the action consists of a free kinetic term plus two F-terms. One term couples bosons to fermions while the other gives screening charges for the bosonic subgroup $\mathrm{GL}(2) \times \mathrm{GL}(2)$. The important consequence is that one can compute the cohomology of the current $G^{-}$in free field theory.

Another application is the relation to type IIB string theory on $\mathrm{AdS}_{3} \times \mathrm{S}^{3} \times \mathrm{T}^{4}$. Here we have shown that string theory is related by our procedure to the $\mathrm{U}(1,1 \mid 2)_{-k} \times \mathrm{U}(1 \mid 1) \times \mathrm{U}(1 \mid 1)$ WZNW model, and that the current $G^{-}$is indeed the standard choice of supersymmetry charge. The current $G^{+}$is a novel choice which is important for our relation.

We suspected a relation between the GL $(N \mid N)$ WZNW models and $\mathcal{N}=(2,2)$ worldsheet supersymmetric theories because the GL(1|1) boundary WZNW model action is very similar to what one finds in boundary superconformal field theories. Knowing the boundary action is an essential aide in solving the model, and hence it was important that we could apply the techniques of superconformal field theory to find boundary actions for our models.

Two tasks remain to be done. Firstly, to use the protected sectors we have found and compute the corresponding cohomology and their correlation functions. It would be particularly interesting to do this for world-sheets of higher genus. Secondly, to verify the boundary actions in an example and use them to compute correlation functions in the boundary theory.

There are also possible generalizations. A topological twist of an $\mathcal{N}=(2,2)$ worldsheet supersymmetric theory has central charge zero, and thus can only correspond to a supergroup WZNW model whose superdimension is zero. We already checked that the B-twist of the superconformal $\mathrm{SL}(2) \times \mathrm{U}(1)$ WZNW model is related to the supergroup SL(2|1) WZNW model, but have not yet discovered other examples. Also note that there exist world-sheet superconformal supergroup WZNW models [34]. One might expect that their B-twists in some cases can be related to WZNW models of orthosymplectic supergroups, and this should be investigated.

\section{Acknowledgments}

We would like to thank Manfred Herbst, Ingo Kirsch, Hubert Saleur, David Ridout and especially Volker Schomerus for helpful discussions.

Open Access. This article is distributed under the terms of the Creative Commons Attribution Noncommercial License which permits any noncommercial use, distribution, and reproduction in any medium, provided the original author(s) and source are credited. 


\section{References}

[1] V. Schomerus and H. Saleur, The GL(1|1) WZW model: From supergeometry to logarithmic CFT, Nucl. Phys. B 734 (2006) 221 [hep-th/0510032] [SPIRES].

[2] H. Saleur and V. Schomerus, On the SU(2|1) WZNW model and its statistical mechanics applications, Nucl. Phys. B 775 (2007) 312 [hep-th/0611147] [SPIRES].

[3] G. Götz, T. Quella and V. Schomerus, The WZNW model on PSU $(1,1 \mid 2)$, JHEP 03 (2007) 003 [hep-th/0610070] [SPIRES].

[4] T. Quella and V. Schomerus, Free fermion resolution of supergroup WZNW models, JHEP 09 (2007) 085 [arXiv:0706.0744] [SPIRES].

[5] T. Creutzig and V. Schomerus, Boundary Correlators in Supergroup WZNW Models, Nucl. Phys. B 807 (2009) 471 [arXiv:0804.3469] [SPIRES].

[6] T. Creutzig and Y. Hikida, Branes in the OSP(1|2) WZNW model, Nucl. Phys. B 842 (2011) 172 [arXiv:1004.1977] [SPIRES].

[7] N.P. Warner, Supersymmetry in boundary integrable models, Nucl. Phys. B 450 (1995) 663 [hep-th/9506064] [SPIRES].

[8] M.R. Gaberdiel and I. Kirsch, Worldsheet correlators in $A d S_{3} / C F T_{2}, J H E P 04$ (2007) 050 [hep-th/0703001] [SPIRES].

[9] A. Dabholkar and A. Pakman, Exact chiral ring of $A d S_{3} / C F T_{2}$, Adv. Theor. Math. Phys. 13 (2009) 409 [hep-th/0703022] [SPIRES].

[10] J. de Boer, J. Manschot, K. Papadodimas and E. Verlinde, The chiral ring of $A d S_{3} / C F T_{2}$ and the attractor mechanism, JHEP 03 (2009) 030 [arXiv:0809.0507] [SPIRES].

[11] N. Berkovits, C. Vafa and E. Witten, Conformal field theory of AdS background with Ramond-Ramond flux, JHEP 03 (1999) 018 [hep-th/9902098] [SPIRES].

[12] C. Candu, T. Creutzig, V. Mitev and V. Schomerus, Cohomological Reduction of $\sigma$-models, JHEP 05 (2010) 047 [arXiv: 1001.1344] [SPIRES].

[13] S. Guruswamy, A. LeClair and A.W.W. Ludwig, gl(N|N) super-current algebras for disordered Dirac fermions in two dimensions, Nucl. Phys. B 583 (2000) 475 [cond-mat/9909143] [SPIRES].

[14] M.R. Zirnbauer, Conformal field theory of the integer quantum Hall plateau transition, hep-th/9905054 [SPIRES].

[15] H. Saleur, Polymers and percolation in two-dimensions and twisted $N=2$ supersymmetry, Nucl. Phys. B 382 (1992) 486 [hep-th/9111007] [SPIRES].

[16] E. Getzler, Manin triples and $N=2$ superconformal field theory, hep-th/9307041 [SPIRES].

[17] P. Di Vecchia, V.G. Knizhnik, J.L. Petersen and P. Rossi, A Supersymmetric Wess-Zumino Lagrangian in Two-Dimensions, Nucl. Phys. B 253 (1985) 701 [SPIRES].

[18] T. Creutzig, Branes in supergroups, arXiv:0908.1816 [SPIRES].

[19] R. Dijkgraaf, H.L. Verlinde and E.P. Verlinde, Notes on topological string theory and 2-D quantum gravity, Based on lectures given at Spring School on Strings and Quantum Gravity, Trieste, Italy, Apr 24-May 2 (1990) and at Cargese Workshop on Random Surfaces, Quantum Gravity and Strings, Cargese, France, May 28-Jun 1 (1990). 
[20] M. Bershadsky, S. Cecotti, H. Ooguri and C. Vafa, Kodaira-Spencer theory of gravity and exact results for quantum string amplitudes, Commun. Math. Phys. 165 (1994) 311 [hep-th/9309140] [SPIRES].

[21] K. Hori et al., Mirror symmetry, American Mathematical Society, Providence U.S.A. (2003).

[22] M. Bershadsky, S. Zhukov and A. Vaintrob, $P S L(n \mid n) \sigma$-model as a conformal field theory, Nucl. Phys. B 559 (1999) 205 [hep-th/9902180] [SPIRES].

[23] T. Quella, V. Schomerus and T. Creutzig, Boundary Spectra in Superspace $\sigma$-models, JHEP 10 (2008) 024 [arXiv:0712.3549] [SPIRES].

[24] A. Giveon, D. Kutasov and N. Seiberg, Comments on string theory on $A d S_{3}$, Adv. Theor. Math. Phys. 2 (1998) 733 [hep-th/9806194] [SPIRES].

[25] J.M. Maldacena and H. Ooguri, Strings in $A d S_{3}$ and $S L(2, R)$ WZW model. I, J. Math. Phys. 42 (2001) 2929 [hep-th/0001053] [SPIRES].

[26] J. Polchinski, String theory. Vol. 2: Superstring theory and beyond, Cambridge University Press, Cambridge U.K. (1998).

[27] I. Brunner, M. Herbst, W. Lerche and B. Scheuner, Landau-Ginzburg realization of open string TFT, JHEP 11 (2006) 043 [hep-th/0305133] [SPIRES].

[28] K. Hori and J. Walcher, D-branes from matrix factorizations, Comptes Rendus Physique 5 (2004) 1061 [hep-th/0409204] [SPIRES].

[29] T. Creutzig, T. Quella and V. Schomerus, Branes in the GL(1|1) WZNW-Model, Nucl. Phys. B 792 (2008) 257 [arXiv:0708.0583] [SPIRES].

[30] T. Creutzig, Geometry of branes on supergroups, Nucl. Phys. B 812 (2009) 301 [arXiv:0809.0468] [SPIRES].

[31] T. Creutzig, T. Quella and V. Schomerus, New boundary conditions for the $c=-2$ ghost system, Phys. Rev. D 77 (2008) 026003 [hep-th/0612040] [SPIRES].

[32] T. Creutzig and P.B. Ronne, The GL(1|1)-symplectic fermion correspondence, Nucl. Phys. B $\mathbf{8 1 5}$ (2009) 95 [arXiv:0812.2835] [SPIRES].

[33] I.T. Ivanov, B.-b. Kim and M. Roček, Complex structures, duality and WZW models in extended superspace, Phys. Lett. B 343 (1995) 133 [hep-th/9406063] [SPIRES].

[34] T. Creutzig, P.B. Ronne and V. Schomerus, $N=2$ Superconformal Symmetry in Super Coset Models, Phys. Rev. D 80 (2009) 066010 [arXiv:0907.3902] [SPIRES]. 